\title{
Species richness of the vascular plants of the Bijagual high Andean forest, Colombia
}

\section{Riqueza de las plantas vasculares del bosque altoandino de Bijagual, Colombia}

\section{Acta Botanica Mexicana}

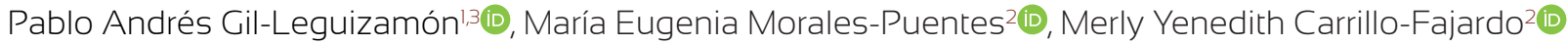

\begin{abstract}
:
Background and Aims: The high Andean forest in Colombia corresponds to a plant community located between 2400-3200(-3500) m a.s.l., characterized by the presence of arboreal species with restricted distributions that determine their physiognomy and composition. Nevertheless, these species have been affected by agricultural activity that has generated a loss of connectivity and richness. This research records the species richness, composition, and distributional data for the vascular flora in the high Andean forest of the Bijagual Massif, Bocayá, Colombia.

Methods: Sampling was carried out in 27 transects. The specimens were organized under the APG IV classification system, curated and deposited in the UPTC herbarium (Universidad Pedagógica y Tecnológica de Colombia). The number of species, list of taxa, distributions for Colombia, and conservation categories according to the IUCN were noted.

Results: We registered 327 species distributed in 151 genera and 69 families; 187 species (56\%) were native to the Andean region, 51 (15\%) were endemic to the Andes and $24(7 \%)$ were endemic to the Eastern cordillera of the Andes, Colombia. Schefflera bejucosa is a new distributional record for Boyacá. Greigia stenolepis, Hieronyma rufa, Puya goudotiana and Tillandsia pallescens are Near Threatened (NT). Plutarchia guascensis, Quercus humboldtii and Symplocos venulosa are classified as Vulnerable (VU), and Diplostephium oblongifolium and Dunalia trianaei as Endangered (EN).

Conclusions: Bijagual is a biological corridor with great potential for biodiversity compared to other high Andean areas in Colombia. Species richness is influenced by other localities near the Massif (Tota and Mampacha), in addition to a possible stochastic and heterogeneous distribution between sampling units. Miconia, Pentacalia, Epidendrum, Elaphoglossum, Tillandsia, and some other genera are species-rich. Clusia alata, Weinmannia balbisiana, Weinmannia rollottii, Ternstroemia cf. camelliifolia, and Brunellia comocladifolia define the arboreal and shrub physiognomy of Bijagual. Key words: Andes, distribution, endemic species, floristic composition, high Andean forest, specific richness.
\end{abstract}

\section{Resumen:}

Antecedentes y Objetivos: El bosque altoandino en Colombia corresponde a una formación vegetal, localizada entre 2400-3200(-3500) m s.n.m. Se caracteriza por la presencia de especies arbóreas con distribución restringida que determinan su fisionomía y composición. No obstante, dichas especies han sido afectadas por la actividad agrícola que ha generado pérdida de conectividad y de riqueza. Esta investigación registra la riqueza, composición y datos de distribución de flora vascular en el bosque altoandino del Macizo de Bijagual, Boyacá, Colombia.

Métodos: Se realizaron muestreos en 27 transectos. Los ejemplares fueron organizados bajo el sistema de clasificación APG IV, curados y depositados en el herbario UPTC (Universidad Pedagógica y Tecnológica de Colombia). Se obtuvo el número de especies, listado de taxa, distribución para Colombia y categorías de conservación según la UICN.

Resultados: Se registraron 327 especies, distribuidas en 151 géneros y 69 familias; 187 especies (56\%) son nativas de la región andina, 51 (15\%) endémicas de los Andes y 24 (7\%) endémicas de la cordillera Oriental colombiana. Schefflera bejucosa es un nuevo registro de distribución para Boyacá. Greigia stenolepis, Hieronyma rufa, Puya goudotiana y Tillandsia pallescens presentan la categoría Casi Amenazado (NT), Plutarchia guascensis, Quercus humboldtii y Symplocos venulosa, Vulnerable (VU); Diplostephium oblongifolium y Dunalia trianaei En Peligro (EN).

Conclusiones: Bijagual es un corredor biológico con gran potencial de biodiversidad comparado con otras áreas altoandinas en Colombia. La riqueza es influenciada por otras localidades cercanas al Macizo (Tota y Mampacha), además de una posible distribución estocástica y heterogénea entre unidades de muestreo. Miconia, Pentacalia, Epidendrum, Elaphoglossum, Tillandsia y algunos otros géneros son ricos en especies. Clusia alata, Weinmannia balbisiana, Weinmannia rollottii, Ternstroemia cf. camelliifolia y Brunellia comocladifolia definen la fisionomia arbórea y arbustiva de Bijagual.

Palabras clave: Andes, bosques altoandinos, composición florística, distribución, especies endémicas, riqueza específica.

${ }^{1}$ Universidad Pedagógica y Tecnológica de Colombia, Facultad de Ingeniería, Grupos SisBio y GISABA, Herbario UPTC, Doctorado en Ciencias Biológicas y Ambientales, Tunja, Colombia.

2Universidad Pedagógica y Tecnológica de Colombia, Facultad de Ciencias, Grupo SisBio, Herbario UPTC, Doctorado en Ciencias Biológicas y Ambientales, Tunja, Colombia.

${ }^{3}$ Author for correspondence: pablo.gil@uptc.edu.co
Received: March 15, 2021.

Reviewed: April 14, 2021.

Accepted by Marie-Stéphanie Samain: November 12, 2021.

Published Online first: November 25, 2021.

Published: Acta Botanica Mexicana 128 (2021).

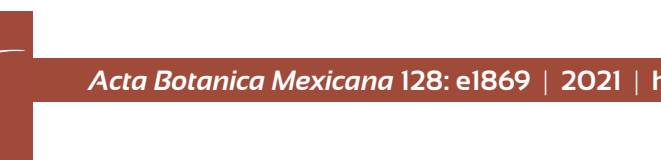

To cite as: Gil-Leguizamón, P. A., M. E. Morales-Puentes and M. Y. Carrillo-Fajardo. 2021. Species richness of the vascular plants of the Bijagual high Andean forest, Colombia. Acta Botanica Mexicana 128: e1869. DOI: http://doi.org/10.21829/abm128.2021.1869 


\section{Introduction}

The high Andean forests are distributed in the Western, Central, and Eastern cordilleras of the Andes in Colombia. The area is located at an altitudinal range of $2400-3500 \mathrm{~m}$ a.s.I. (Rangel-Ch. et al., 1997). However, floristic research has occurred mainly in the Western and Central cordilleras (Mosquera-Ramos et al., 2007; León et al., 2009; Idárraga and Callejas, 2011; Alzate et al., 2013; David et al., 2014; Ramírez-Padilla et al., 2015). Existing research in the Eastern cordillera is focused on distributional patterns and dominance of plant communities (Marín and Betancur, 1997; Franco-R. and Betancur, 1999; Galindo et al., 2003; Cortés, 2003; Arias and Barrera, 2007; Fernández-A. and Hernández-S., 2007).

The high Andean forests are characterized by the presence of biogeographically restricted elements that define the physiognomy and composition of the forests (Rangel-Ch. et al., 1997). The forests are very humid and rich in woody and herbaceous plants. Because of these characteristics, species composition is specific at local levels in the Eastern cordillera (Van der Hammen and Cleef, 1983).

The high Andean forests have had the constant influence of farming (especially of potatoes, onions, apples, pears, and peaches), ungulate-grazing (cattle, horses, and pigs), and mining (coal, emeralds, and iron). It also has lost areas to a matrix of grasslands (Pennisetum Rich., Brachiaria (Trin.) Griseb., and Holcus lanatus L.), and this has led to a loss not only of forest biodiversity, but also of connectivity (Armenteras et al., 2003, 2013; Etter et al., 2006; Medina et al., 2015).

The objective of this research is presenting the description of the composition, richness and distribution of species of the high Andean forest of the Bijagual Massif, Boyacá, Colombia. Additionally, we have included data of threatened categories and endemic species.

\section{Materials and Methods}

\section{Study area}

The Bijagual Massif is located in the department of Boyacá, Colombia, at the coordinates $5^{\circ} 26^{\prime} 10.28^{\prime \prime}-5^{\circ} 15^{\prime} 40.21^{\prime \prime} \mathrm{N}$ and $73^{\circ} 13^{\prime} 5.20^{\prime \prime}-73^{\circ} 21^{\prime} 58.19^{\prime \prime} \mathrm{W}$, with an area of extension of 8604 ha (Gil-Leguizamón et al., 2020). Its limits are the Tota lake to the north and the Mamapacha Massif to the south (Tota-Bijagual-Mamapacha complex; Morales et al., 2007). The high Andean forest distribution (2682-3268 m a.s.I.) abuts paramo flora at the highest altitudes (2990$3460 \mathrm{~m}$ a.s.I.). Currently, Bijagual is not a protected natural area. There are excessive activities of inappropriate soil use with more than $50 \%$ committed to farming and cattle (Fig. 1; Gil, 2016).

\section{Data collection}

We established 27 transects in the high Andean forest of Bijagual $\left(100 \times 5 \mathrm{~m}=500 \mathrm{~m}^{2}\right)$ (Rangel-Ch. and Velásquez, 1997). For each individual, the abundance, growth form, height, coverage, diameter at breast height for trees, and basal area for shrubs were noted (data published in Gil-Leguizamón et al., 2020).

For determination of botanical material, specialized literature for families and genera of the woody plants (Gentry, 1993), Flora de Colombia (FDC, 2021), Flora Neotropica (SFNM, 2021), Flora de Costa Rica (BMCR, 2021), and Flora de Panamá (PAC, 2021) were used, as well as the International Plant Name Index (IPNI, 2021) for correct citations of the scientific names; for suprafamiliar classification APG IV (2016) was used. Each specimen was confirmed with the HECASA (Universidad de Pamplona, Colombia) and UPTC (Universidad Pedagógica y Tecnológica de Colombia) herbarium collections, virtual herbarium reviews (COL herbarium; COL, 2021), and web pages (Botanicus, 2021; TPL , 2021; Tropicos, 2021) and with a botanical specialist. Vouchers were deposited in the UPTC herbarium reference collection under Gil-Leguizamón P.A. collection numbers.

\section{Data analysis}

This study of vascular plants involved the families and genera with high species richness. For each species, distributional data were obtained in Colombia (of endemic and native species) according to Bernal et al. (2016). We present the table in order to corroborate new records and species identification with their conservation category (IUCN, 2021). The results presented here expand the information of Gil-Leguizamón et al. (2020) about the structure and diversity of the vegetation of the Bijagual Massif paramo complex. 


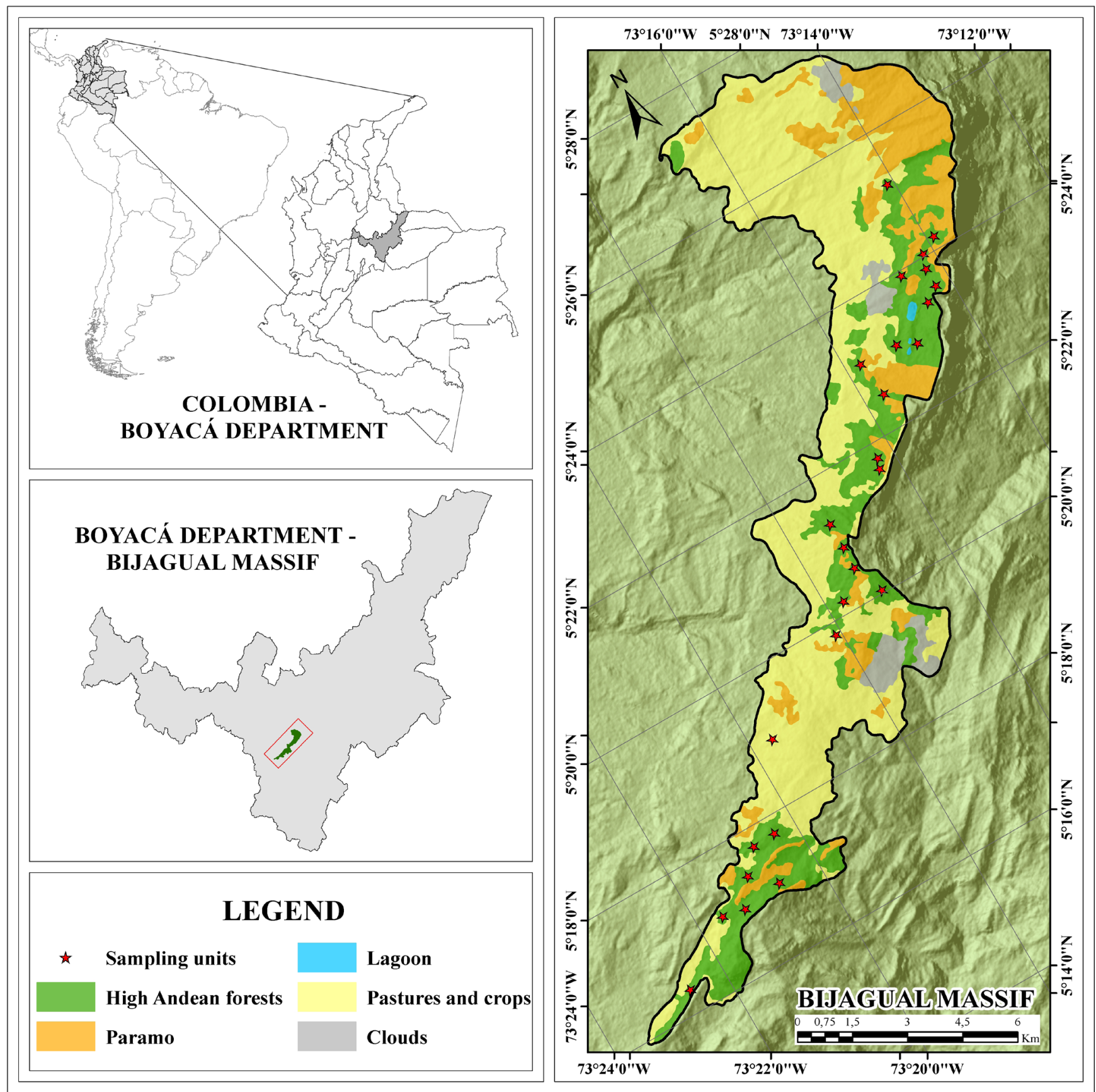

Figure 1: Biological corridor of Bijagual Massif, Boyacá, Colombia.

\section{Results}

We recorded 327 species, distributed in 151 genera and 69 families (Appendix; Figs. 2, 3, 4, 5, 6, 7, 8, 9 show some of these species). The families with the highest species richness (16 families or 23\%) are: Asteraceae, Orchidaceae, Melastomataceae, Ericaceae, Bromeliaceae, Rubiaceae, Poaceae, Rosaceae and Araliaceae. These contained $59 \%$ of the genera and $70 \%$ of the species (Table). The most diverse genera are (16 or 11\%) Miconia Ruiz \& Pav.,
Pentacalia Cass., Epidendrum L., Tillandsia L., Ageratina Spach, Diplostephium Kunth, Stelis Sw., Pleurothallis R. Br., Rubus L., and represent $35 \%$ of the total number of species (Table).

Twenty percent of the families (14 families) are represented by three or four species and $57 \%$ (39 families) by one or two. Of the genera, 13\% (20 genera) have three or four species, and $76 \%$ (117 genera) one or two taxa, respectively (Appendix). 


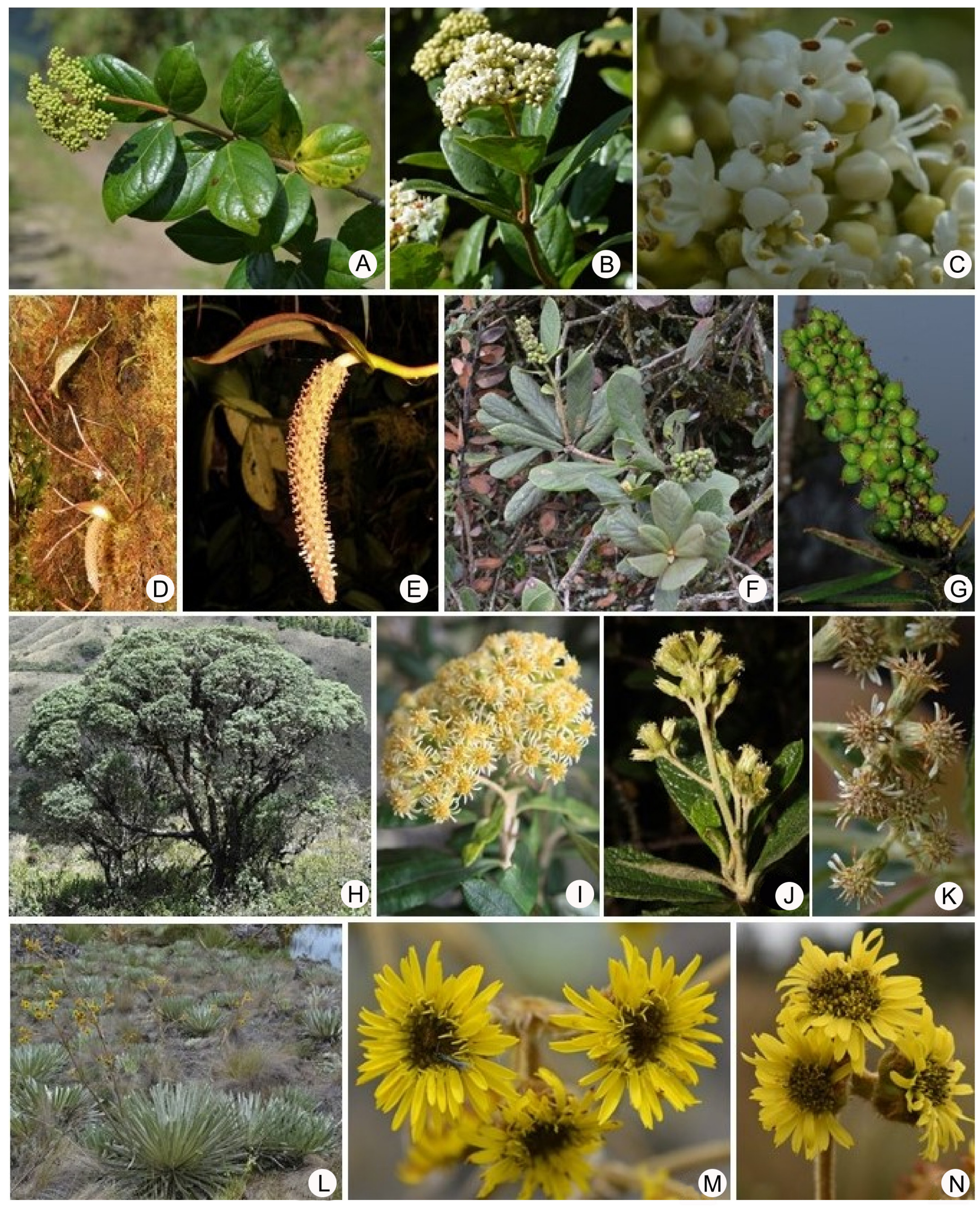

Figure 2: Recorded vascular plants in the Bijagual Massif, Boyacá, Colombia. A-C. Viburnum triphyllum Benth.; D, E. Anthurium sp.; F, G. Oreopanax mutisianus (Kunth) Decne. \& Planch.; H, I. Diplostephium floribundum (Benth.) Wedd.; J, K. Diplostephium tenuifolium Cuatrec.; L-N. Espeletia boyacensis Cuatrec. 

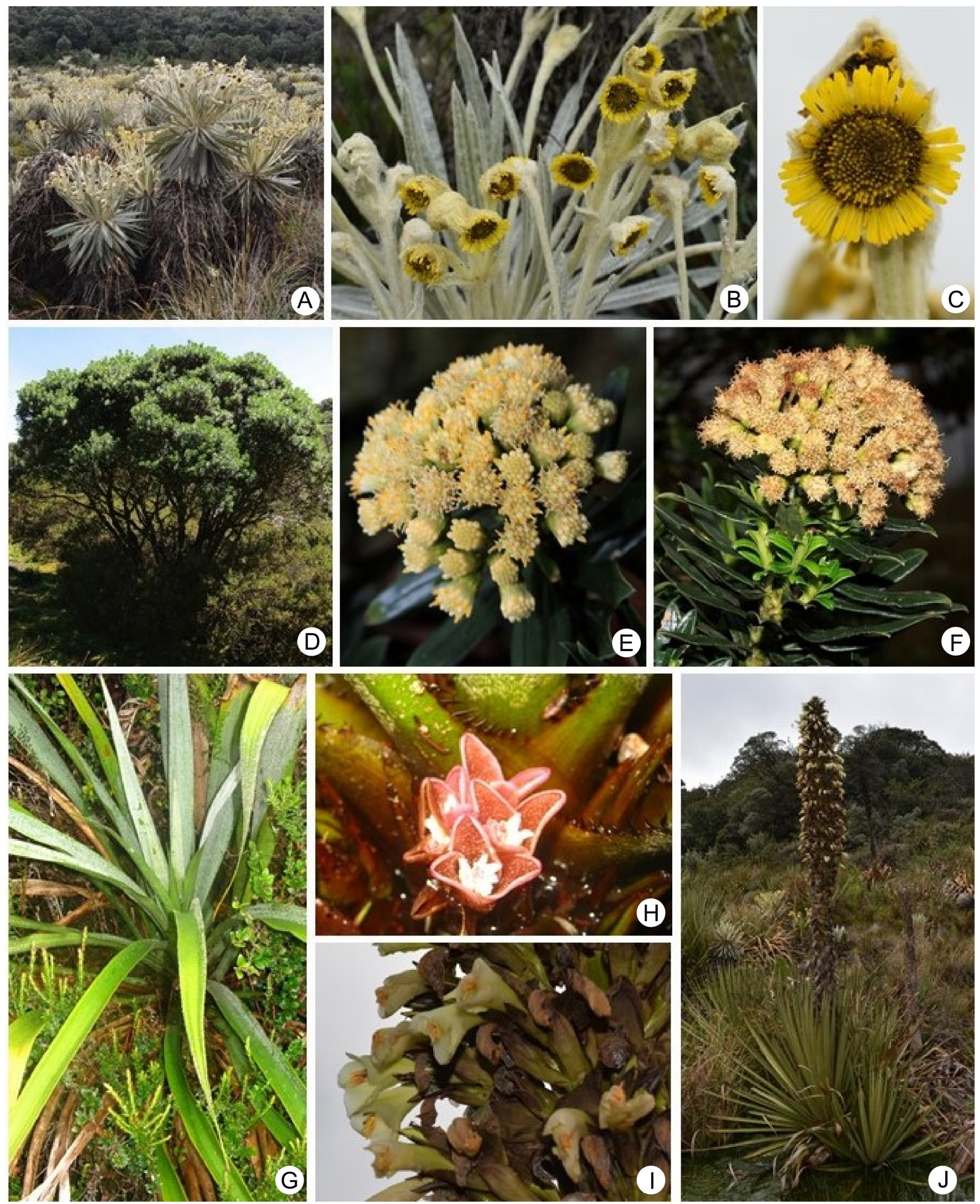

Figure 3: Recorded vascular plants in the Bijagual Massif, Boyacá, Colombia. A-C. Espeletia murilloi Cuatrec.; D-F. Pentacalia pulchella (Kunth) Cuatrec.; G, H. Greigia stenolepis L.B. Sm.; I, J. Puya goudotiana Mez. 

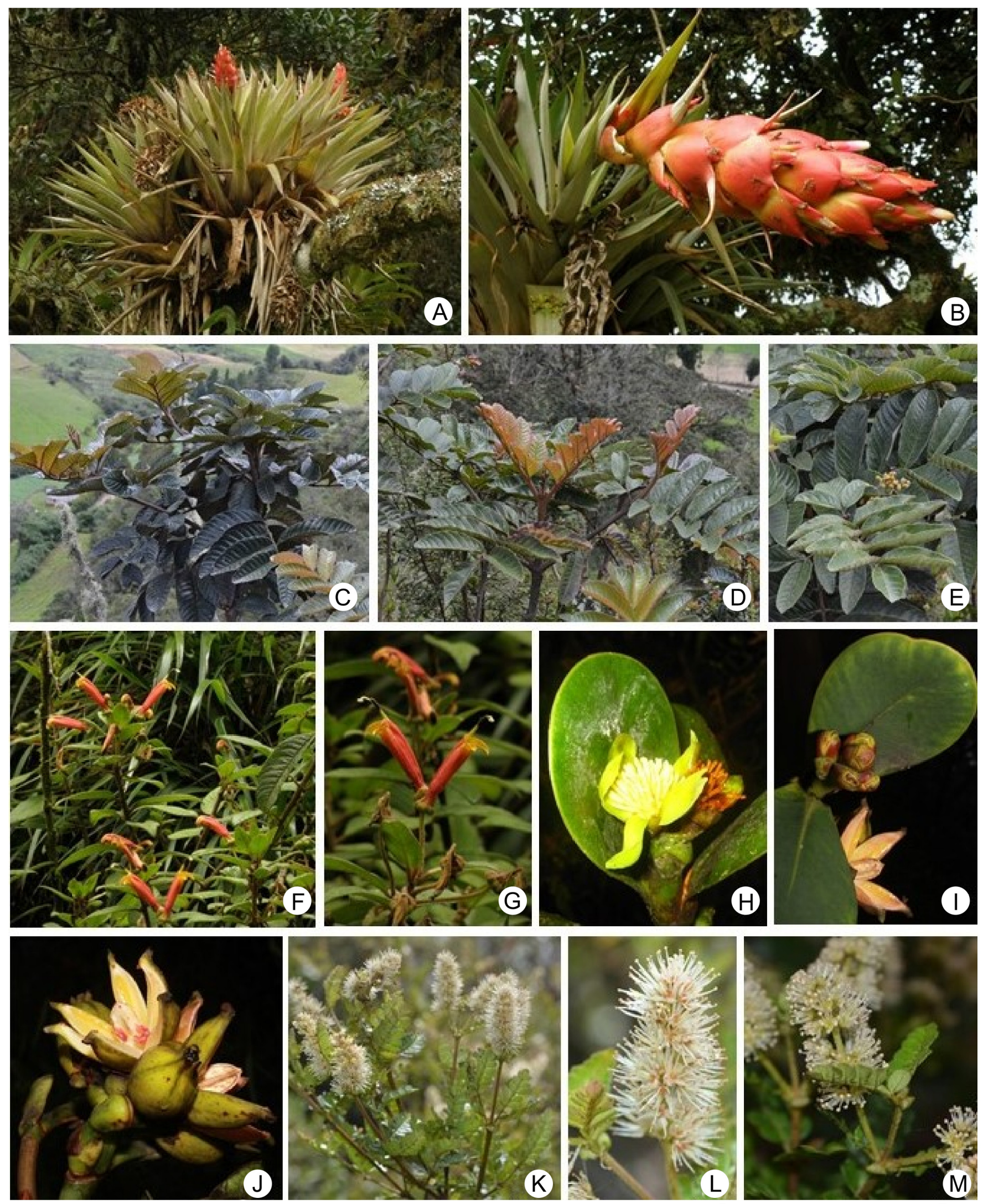

Figure 4: Recorded vascular plants in the Bijagual Massif, Boyacá, Colombia. A, B. Tillandsia turneri Baker; C-E. Brunellia propinqua Kunth; F, G. Siphocampylus sp.; H-J. Clusia multiflora Kunth; K-M. Weinmannia fagaroides Kunth. 

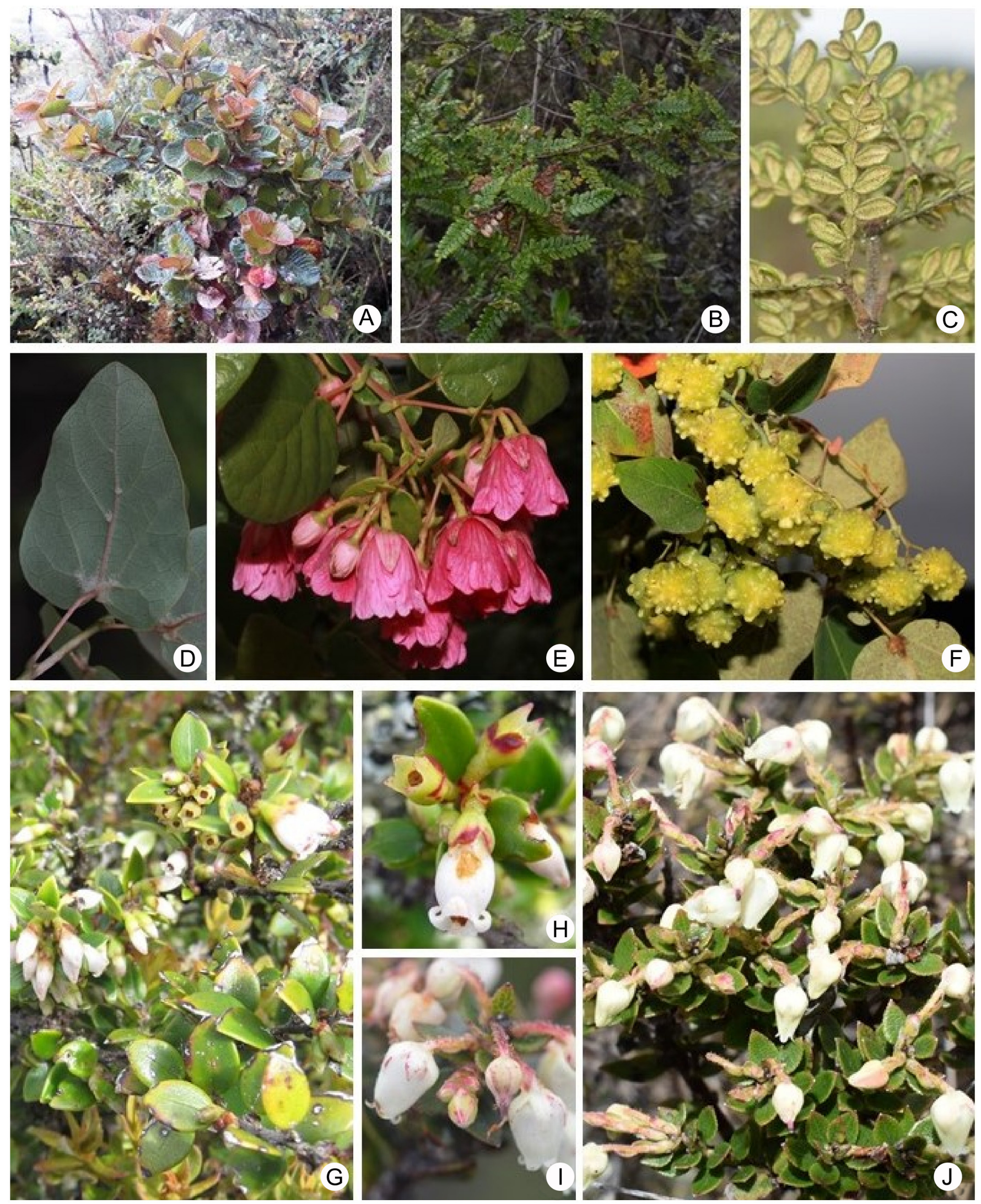

Figure 5: Recorded vascular plants in the Bijagual Massif, Boyacá, Colombia. A. Weinmannia rollottii Killip; B, C. Weinmannia tomentosa L. f.; D-F. Vallea stipularis L. f.; G, H. Disterigma alaternoides (Kunth) Nied.; I, J. Gaultheria anastomosans (L. f.) Kunth. 

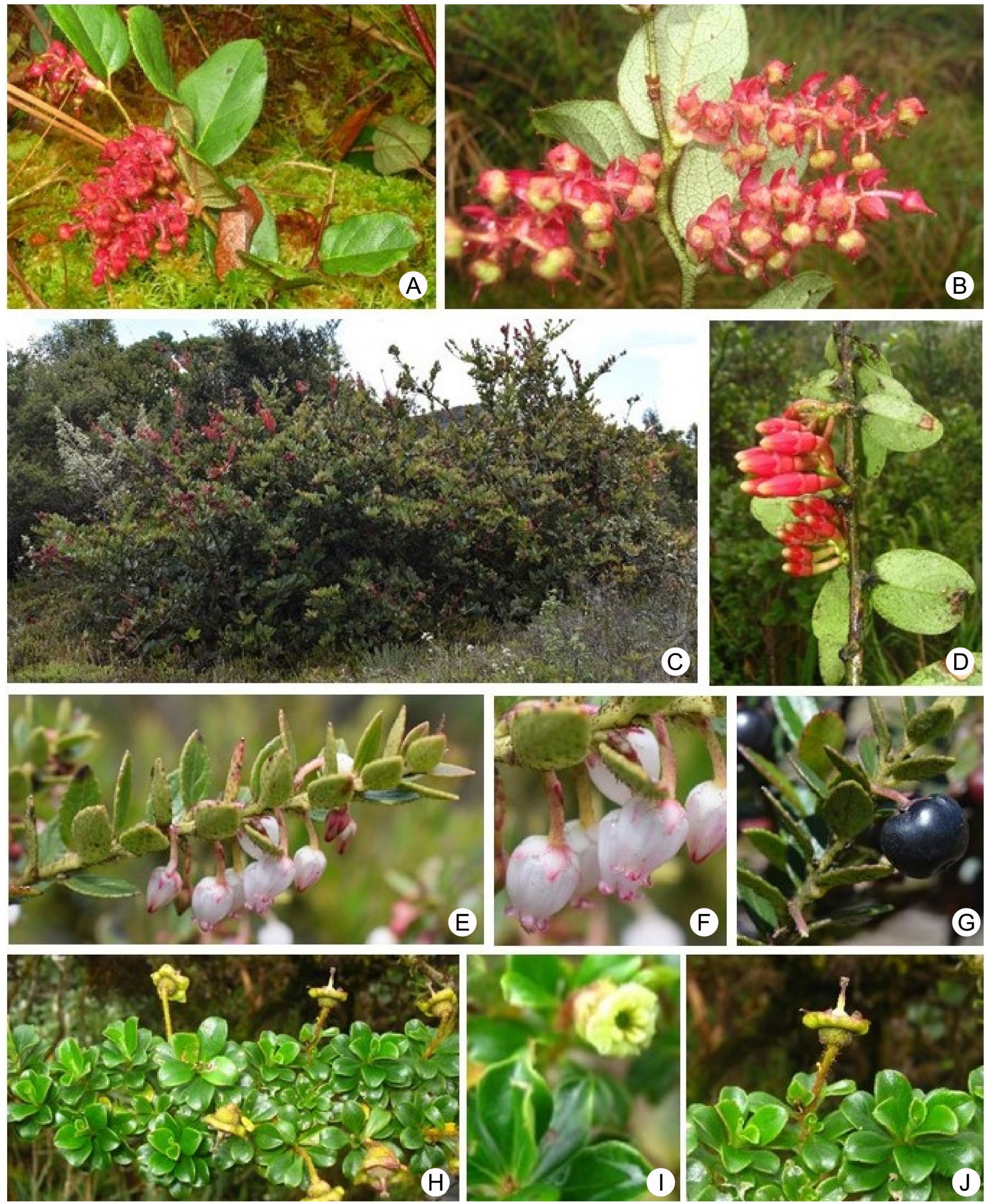

Figure 6: Recorded vascular plants in the Bijagual Massif, Boyacá, Colombia. A, B. Gaultheria erecta Vent.; C, D. Macleania rupestris (Kunth) A.C. Sm.; E-G. Pernettya prostrata (Cav.) DC.; H-J. Escallonia myrtilloides L. f. 

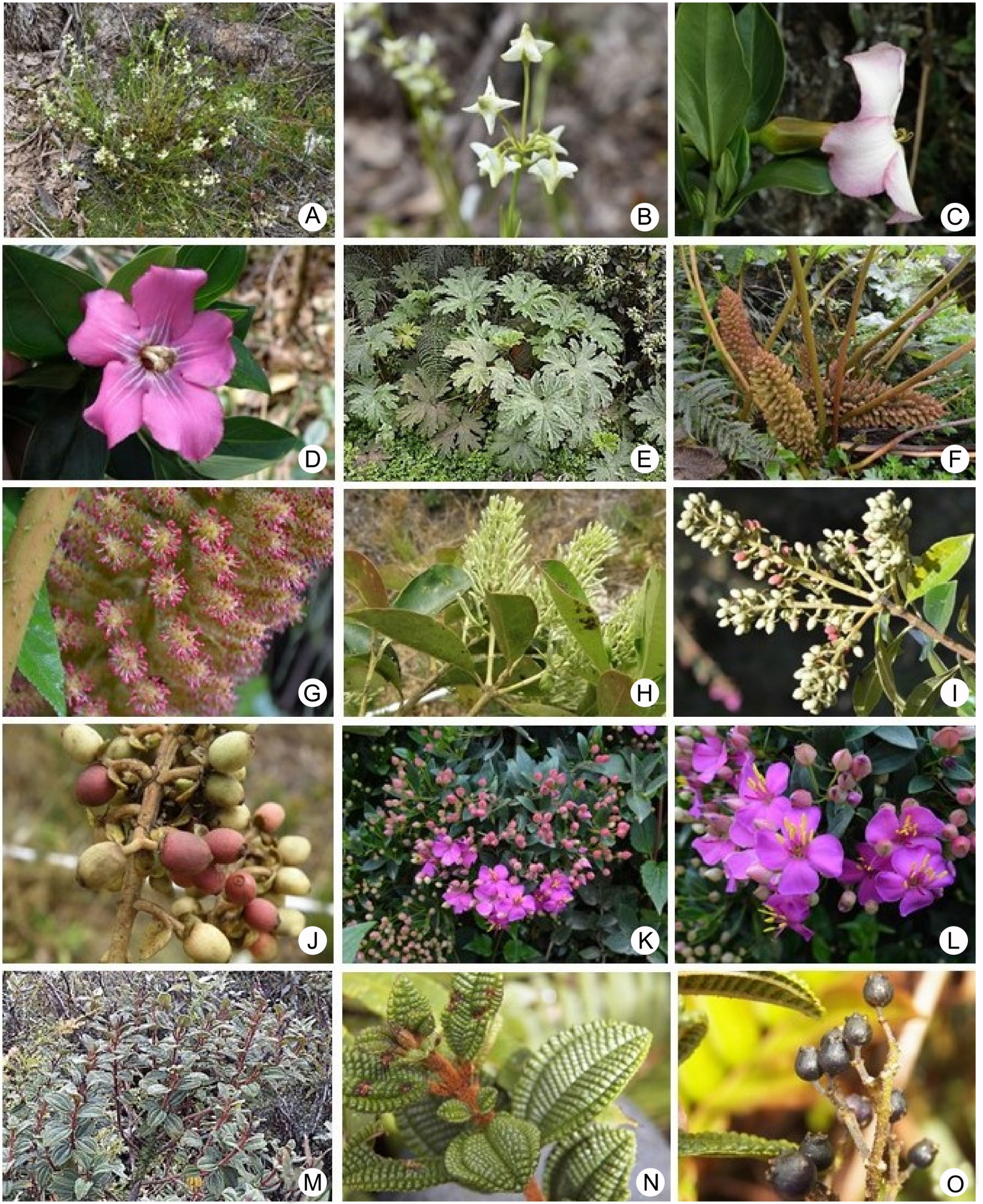

Figure 7: Recorded vascular plants in the Bijagual Massif, Boyacá, Colombia. A, B. Halenia asclepiadea (Kunth) G. Don; C, D. Symbolanthus calygonus (Ruiz \& Pav.) Griseb. ex Gilg; E-G. Gunnera cf. schultesii L.E. Mora; H-J. Gaiadendron punctatum (Ruíz \& Pav.) G. Don; K, L. Bucquetia glutinosa (L. f.) DC.; M-O. Miconia cleefii L. Uribe. 

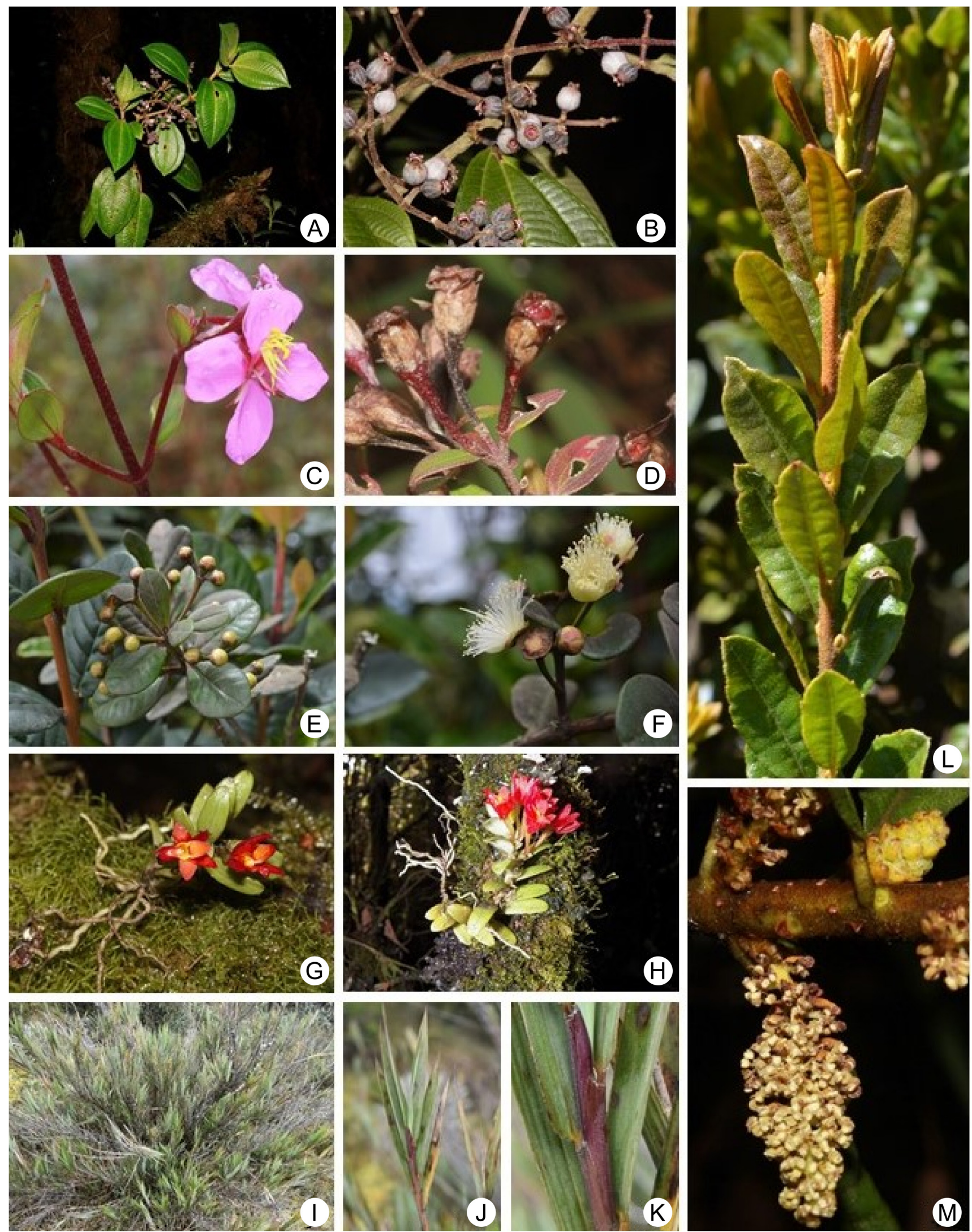

Figure 8: Recorded vascular plants in the Bijagual Massif, Boyacá, Colombia. A, B. Miconia stipularis Naudin; C, D. Monochaetum myrtoideum Naudin; E, F. Myrcianthes rhopaloides (Kunth) McVaugh; G, H. Fernandezia lanceolata (L.O. Willams) Garay \& Dunst.; I-K. Chusquea tessellata Munro; L, M. Morella parvifolia (Benth.) Parra-Os. 

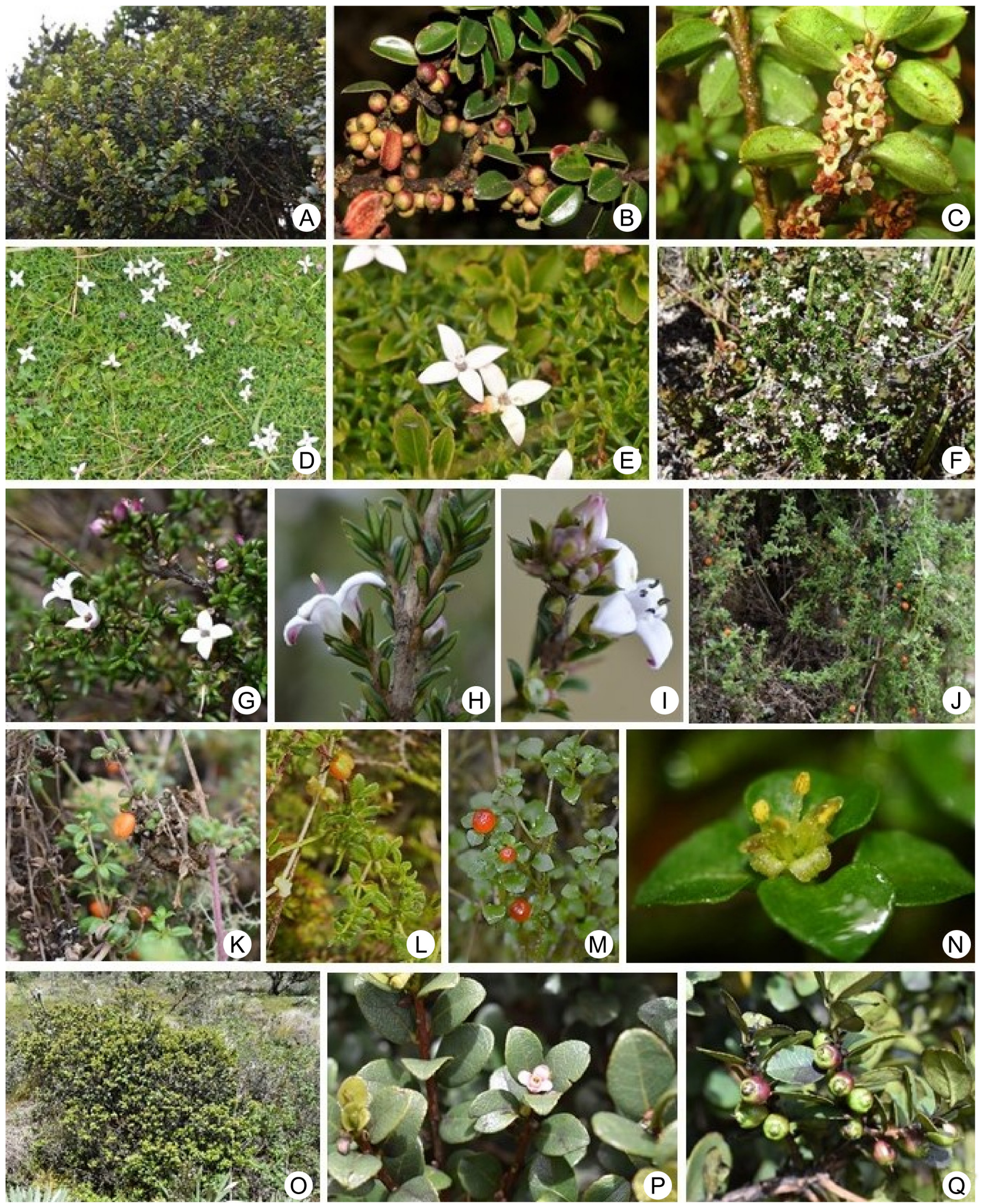

Figure 9: Recorded vascular plants in the Bijagual Massif, Boyacá, Colombia. A-C. Myrsine dependens (Ruiz \& Pav.) Spreng.; D, E. Arcythophyllum muticum (Wedd.) Standl.; F-I. Arcythophyllum nitidum (Kunth) Schltdl.; J-L. Galium hypocarpium (L.) Endl. ex Griseb.; M, N. Nertera granadensis (Mutis ex L. f.) Druce; O-Q. Symplocos theiformis (L. f.) Oken. 
Table: Most representative families and genera in the Bijagual Massif, Boyacá, Colombia.

\begin{tabular}{|c|c|c|c|c|c|}
\hline $\mathbf{N}^{\circ}$ & Families & Genera & Species & Genera & Species \\
\hline 1 & Asteraceae & 14 & 47 & Miconia Ruiz \& Pav. & 15 \\
\hline 2 & Orchidaceae & 13 & 42 & Pentacalia Cass. & 12 \\
\hline 3 & Melastomataceae & 6 & 22 & Epidendrum L. & 10 \\
\hline 4 & Ericaceae & 10 & 21 & Tillandsia $\mathrm{L}$. & 9 \\
\hline 5 & Bromeliaceae & 7 & 19 & Ageratina Spach & 8 \\
\hline 6 & Rubiaceae & 6 & 12 & Diplostephium Kunth & 7 \\
\hline 7 & Poaceae & 6 & 11 & Stelis Sw. & 7 \\
\hline 8 & Rosaceae & 4 & 8 & Pleurothallis R. Br. & 6 \\
\hline 9 & Araliaceae & 3 & 8 & Palicourea Aubl. & 6 \\
\hline 10 & Solanaceae & 5 & 7 & Rubus L. & 5 \\
\hline 11 & Cyperaceae & 4 & 7 & Anthurium Schott & 5 \\
\hline 12 & Piperaceae & 2 & 6 & Oreopanax Decne. \& Planch. & 5 \\
\hline 13 & Lauraceae & 4 & 5 & Weinmannia L. & 5 \\
\hline 14 & Primulaceae & 3 & 5 & Disterigma (Klotzsch) Nied. & 5 \\
\hline 15 & Araceae & 1 & 5 & Gaultheria L. & 5 \\
\hline 16 & Cunoniaceae & 1 & 5 & Lepanthes Sw. & 5 \\
\hline Represer & families: 16 (23\%) & $89(59 \%)$ & $230(70 \%)$ & Representative genera: $16(11 \%)$ & $115(35 \%)$ \\
\hline \multicolumn{2}{|c|}{ Other families: 53 (77\%) } & $62(41 \%)$ & $97(30 \%)$ & Other genera: 135 (89\%) & $212(65 \%)$ \\
\hline \multicolumn{2}{|c|}{ Total families: 69 (100\%) } & $151(100 \%)$ & $327(100 \%)$ & Total genera: $151(100 \%)$ & $327(100 \%)$ \\
\hline
\end{tabular}

One hundred eighty-seven species (56\%) are native to the Andean region, and $51(15 \%)$ are endemic to the Andes from this area. Only 24 (7\%) are endemic to the Colombian Eastern cordillera. Passiflora adulterina L. f., and Plutarchia guascensis (Cuatrec.) A.C. Sm. are restricted to the Cundinamarca-Boyacá highlands. The latter two species are also recorded in the departments of Quindío and Santander (Bernal et al., 2016, Appendix). Schefflera bejucosa Cuatrec. (Araliaceae) is a new record for the department of Boyacá. According to the IUCN (2021) records and the Colombian Red Books, 78 species do not have information about their conservation assessment and threat categorization, but 151 taxa are categorized as Not Evaluated (NE) and 89 as Least Concern (LC) (García et al., 2005; Betancur and García, 2006; Hernández and García, 2006; Bernal et al., 2016). Greigia stenolepis L.B. Sm. (Fig. 3: G, H), Hieronyma rufa P. Franco, Puya goudotiana Mez (Fig. 3: I, J) and Tillandsia pallescens Betancur \& García Nestor are Near Threatened (NT) (Betancur and García, 2006).
Plutarchia guascensis (Cuatrec.) A.C. Sm., Quercus humboldtii Bonpl. and Symplocos venulosa Cuatrec. are classified as Vulnerable (VU) (Cárdenas and Salinas, 2007), and Diplostephium oblongifolium Cuatrec., and Dunalia trianaei Dammer as Endangered (EN).

\section{Discussion}

The study area comprised 1.35 ha and overlapped with a minimum unit (0.1 ha) (Gentry, 1982, 1995; Rangel-Ch. and Velásquez, 1997). We determined that the Bijagual flora has great biodiversity potential as a biological corridor. The species richness and composition in Bijagual is higher compared to other localities in the Colombian Eastern cordillera (Avella-M. et al., 2014; Gil, 2016; Carrillo et al., 2017; Gil-Leguizamón et al., 2020).

In 0.1 ha the average estimated species richness is 109 spp. \pm 17 (some of the species are shown here (Figs. $2,3,4,5,6,7,8,9)$, so the results suggest that the number of taxa was very high compared to that reported from 
other high Andean forests. Species richness fluctuates between 43 and 53 species in other forests in the same cordillera (Marín and Betancur, 1997; Galindo et al., 2003; Arias and Barrera, 2007) and between 18 and 69 species for forests in the Central and Western cordilleras (Gentry, 1995; León et al., 2009; Alvear et al., 2010; Bohórquez et al., 2011; Abud-H. and Torres, 2016; Segura-Madrigal et al., 2019).

Our study found that the total floral richness (327 spp.) is greater for Bijagual than that recorded by Carvajal et al. (2014). These authors determined for the same area 275 species in 86 families. This result is influenced by the total assessed area including species with trunk diameters $\leq$ to $2.5 \mathrm{~cm}$. This criterion extends the richness to $152 \mathrm{spe}-$ cies (39\%). Characteristics that contribute to the species richness are strongly associated with the flora from the Tota corridor (north) and Mamapacha (south) in the altitudinal and latitudinal gradients. This is possibly a stochastic species distribution with high species replacement between sampling units (Gil-Leguizamón et al., 2020).

The Bijagual high Andean forests have typical floristic elements of rocky formations (Cuatrecasas, 1934; Rangel-Ch., 1995; Arias and Barrera, 2007). Alvear et al. (2010) and Carvajal et al. (2014) recorded the families Asteraceae, Ericaceae, Melastomataceae, Rubiaceae, Cunoniaceae, and Poaceae as main groups that characterize the ecosystem, which is consistent with the data obtained in this study. However, the Bijagual flora is also represented by families such as Orchidaceae, Rosaceae, Solanaceae, Cyperaceae, Bromeliaceae, and Araliaceae. These make up 29\% (95 species) of the total assessed area.

Genera such as Miconia, Pentacalia, Epidendrum, Elaphoglossum Schott ex J. Sm., Tillandsia, Ageratina, Diplostephium, Weinmannia L., Stelis, and Hymenophyllum $\mathrm{Sm}$. are representative in Bijagual and they are diverse (Table). These are recorded by Cuatrecasas (1958), Marín and Betancur (1997), Franco-R. and Betancur (1999), Fernández-A. and Hernández-S. (2007), Montenegro and Vargas (2008), Álvaro-Fajardo et al. (2013) and Jadán et al. (2017), for Colombian and Ecuadorian high mountain formations. Likewise, the abovementioned authors included Brunellia Ruiz \& Pav. (Figs. 4C-E), Clusia L. (Figs. 4H-J), Drimys J.R. Forst. \& G. Forst., Oreopanax Decne. \& Planch., Geissan- thus Hook. f., Palicourea Aubl., Piper L., Escallonia Mutis ex L. f., Symplocos Jacq. (Figs. 90-Q), Hesperomeles Lindl., Clethra L., Ilex L., and Ocotea Aubl., which are also recorded with low species richness in this research (Figs. 2, 3, 4, 5, $6,7,8,9)$.

In the high Andean Eastern cordillera, records of Lauraceae (Ocotea and Persea Mill.) and Melastomataceae (Axinea A. Juss., Tibouchina Aubl., Bucquetia DC., and Miconia) are dominant in the arboreal and shrubby strata and are also registered for Bijagual, with species like Clusia alata Planch. \& Triana, C. elliptica Kunth and C. multiflora Kunth (Figs. 4H-J) (Clusiaceae), Weinmannia balbisiana Kunth, W. fagaroides Kunth (Figs. 4K-M), W. reticulata Ruiz \& Pav., W. rollottii Killip (Fig. 5A), W. tomentosa L. f. (Figs. 5B, C) (Cunoniaceae), Ternstroemia cf. camelliifolia Linden \& Planch. (Pentaphylacaceae), Brunellia cf. comocladifolia Bonpl. and B. propinqua Kunth (Figs. 4C-E) (Brunelliaceae) (Gentry, 2001; Avella-M. et al., 2014).

Between the altitudes of 2900-3000(-3200) m, the families Ericaceae and Primulaceae (Myrsine L. and Geissanthus), as well as the genera Oreopanax, Weinmannia, Hedyosmum Sw., Viburnum L., Vallea Mutis ex L. f., Clusia, Gaiadendron G. Don, Myrica L., Piper, Hesperomeles, Palicourea, and Muehlenbeckia Meisn. are recorded (Gentry, 2001; Galindo et al., 2003; Olaya-Angarita et al., 2019; and Gil-Leguizamón et al., 2020). Similarly, some genera recorded in high Andean forest include species that are typical of the forest-paramo transitions in Bijagual, such as Gaiadendrum punctatum (Ruíz \& Pav.) G. Don. (Figs. 7H-J), Hesperomeles obtusifolia (Pers.) Lindl., Weinmannia fagaroides (Figs. 4K-M) and W. balbisiana.

Species from subparamo and paramo ecosystems were recorded in the high Andean forest due to vertical migration, although they do not belong to the actual forest (Rangel-Ch., 2000). Suitable features for colonization and development are found at lower altitudes (paramo colonization; Van der Hammen, 1998). Special cases are Hypericum lycopodioides Triana \& Planch., Arcythophyllum nitidum (Kunth) Schltdl., Puya goudotiana Mez, Paepalanthus columbiensis Ruhland, Espeletia murilloi Cuatrec., and E. boyacensis Cuatrec., which are also documented by Cortés (2003) for the Eastern cordillera and by Alvear et al. (2010) for the Central cordillera in Colombia. 


\section{Author contributions}

PAG, MEM, and MYC conceived and designed the study, performed the analyses, contributed to data acquisition and interpretation, and wrote the manuscript. All authors contributed to the discussion, review, and approval of the final manuscript.

\section{Funding}

This study was supported by Ecopetrol S.A., the Universidad Pedagógica y Tecnológica de Colombia - UPTC and the Biological Systematic Research group, through the Ecopetrol S.A. and Ecopetrol-UPTC agreement No. DHS 5211071.

\section{Acknowledgements}

Thanks to herbaria COL, HECASA and UPTC for access to their reference collections. We also thank some essential persons whose contributions solidified this research: C.N. Díaz-Pérez, J.E. Gil-Novoa, W.J. Bravo, N.T. Manrique, V.M. Alvarado, A.L. Simbaqueba, C. Castro, O. Garzón, D.A. Moreno, D. Torres, Sistemática Biológica members, for the hard field and laboratory work; and the researchers D.A. Giraldo-Cañas, L.R. Sánchez, and M.A. Quijano-Abril for the identification of botanical material.

\section{Literature cited}

Abud-H., M. and A. M. Torres. 2016. Caracterización florística de un bosque alto andino en el Parque Nacional Natural Puracé, Cauca, Colombia. Boletín Científico del Centro de Museos, Museo de Historia Natural 20(1): 27-39.

Álvaro-Fajardo, V. M., M. E. Morales-Puentes and E. F. LarrotaEstupiñan. 2013. Bromeliaceae en algunos municipios de Boyacá y Casanare, Colombia. Revista de la Academia Colombiana de Ciencias Exactas Físicas y Nautrales 37(142): 5-18.

Alvear, M., J. Betancur and P. Franco-Roselli. 2010. Diversidad florística y estructura de remanentes de bosque andino en la zona de amortiguación del Parque Nacional Natural los Nevados, cordillera Central colombiana. Caldasia 32(1): 3963.

Alzate, F., A. Idárraga, O. Díaz and W. Rodríguez. 2013. Flora de los bosques montanos de Medellín. Programa expedición de Antioquia-2013, Series Biodiversidad y Recursos Naturales. Medellín, Colombia. 552 pp.
APG IV. 2016. An update of the Angiosperm Phylogeny Group classification for the orders and families of flowering plants: The Angiosperm Phylogeny Group. Botanical Journal of the Linnean Society 181: 1-20. DOI: https://doi.org/10.1111/ boj.12385

Arias, M. A. and J. Barrera. 2007. Caracterización florística y estructural de la vegetación vascular en áreas con diferente condición de abandono en la cantera Soratama, localidad de Usaquén, Bogotá. Universitas Scientiarum 12: 25-45.

Armenteras, D., F. Gast and H. Villareal. 2003. Andean forest fragmentation and the representativeness of protected natural areas in the eastern Andes, Colombia. Biological Conservation 113(2): 245-256. DOI: https://doi. org/10.1016/s0006-3207(02)00359-2

Armenteras, D., E. Cabrera, N. Rodríguez and J. Retana. 2013. National and regional determinants of tropical deforestation in Colombia. Regional Environmental Change 13(6): 1181-1193. DOI: https://doi.org/10.1007/s10113013-0433-7

Avella-M. A., S. Torres-R., W. Gómez-A. and M. Pardo-P. 2014. Los páramos y bosques altoandinos del pantano de Monquentiva o pantano de Martos (Guatavita, Cundinamarca, Colombia): caracterización ecológica y estado de conservación. Biota Colombiana 15(1): 3-39.

Bernal, R., R. Gradstein and M. Celis. 2016. Catálogo de plantas y líquenes de Colombia. Facultad de Ciencias, Instituto de Ciencias Naturales, Universidad Nacional de Colombia. Bogotá, Colombia. 3060 pp.

Betancur, J. and N. García. 2006. Las bromelias. In: García, N. and G. Galeano (eds.). Libro rojo de plantas de Colombia 3: Las bromelias, las labiadas y las pasifloras. Instituto Alexander von Humboldt - Instituto de Ciencias Naturales de la Universidad Nacional de Colombia - Ministerio de Ambiente, Vivienda y Desarrollo Territorial. Bogotá, Colombia. Pp. 51-384.

BMCR. 2021. Biblioteca "Héctor Gamboa Paniagua" Museo de Costa Rica (BMCR). San José, Costa Rica. http://biblioteca. museocostarica.go.cr/ (consulted October, 2021).

Bohórquez, A. F., D. Sanín and N. Walter-Silva. 2011. Estructura y composición arbórea de los bosques del diablo (San Felix, Salamina, Caldas), selva altoandina de la cordillera Central colombiana. Boletín Científico del Centro de Museos, Museo de Historia Natural 16(2): 39-52. 
Botanicus. 2021. Botanicus Digital Library. Missouri Botanical Garden. Saint Louis, USA. www.botanicus.org (consulted October, 2021).

Cárdenas, D. and N. Salinas. 2007. Libro rojo de plantas de Colombia, Vol. 4: Especies maderables amenazadas, primera parte. Serie de libros rojos de especies amenazadas en Colombia, Instituto Amazónico de Investigaciones Científicas (SINCHI) - Ministerio de Ambiente, Vivienda y Desarrollo Territorial. Bogotá, Colombia. 232 pp.

Carrillo, M. Y., M. E. Morales-Puentes and P. A. Gil-Leguizamón. 2017. Catálogo de la flora (angiospermas) del páramo de Bijagual, Boyacá, Colombia. Boletín Científico del Centro de Museos 21(2): 15-29.

Carvajal, R. L., W. Ariza, L. Caro and N. Valero. 2014. Especies forestales representativas del suroriente de Boyacá. Árboles de CORPOCHIVOR - Corporación Autónoma Regional de Chivor (CORPOCHIVOR). Bogotá, Colombia. 543 pp.

COL. 2021. Herbario Nacional Colombiano. Universidad Nacional de Colombia, Bogotá, Colombia. http://www.biovirtual. unal.edu.co/es/colecciones/search/plants/ (consulted October, 2021).

Cortés, S. 2003. Estructura de la vegetación arbórea y arbustiva en el costado oriental de la serranía de Chía (CundinamarcaColombia). Caldasia 25(1): 119-137.

Cuatrecasas, J. 1934. Observaciones geobotánicas en Colombia. Trabajos del Museo Nacional de Ciencias Naturales Serie Botánica 27: 1-144.

Cuatrecasas, J. 1958. Aspectos de la vegetación natural en Colombia. Revista de la Academia Colombiana de Ciencias, Exactas, Físicas y Naturales 10(40): 221-268.

David, H., O. Díaz, L. M. Urrea and F. Cardona. 2014. Guía ilustrada del cañón del río Porce, Antioquia. Empresas Públicas de Medellín (EMP), Herbario Universidad de Antioquia, Universidad de Antioquia. Medellín, Colombia. 264 pp.

Etter, A., C. McAlpine, K. Wilson, S. Phinn and H. Possingham. 2006. Regional patterns of agricultural land use and deforestation in Colombia. Agriculture, Ecosystems \& Environment 114(2-4): 369-386. DOI: https://doi. org/10.1016/j.agee.2005.11.013

FDC. 2021. Flora de Colombia (FDC). Universidad Nacional de Colombia. Bogotá, D.C., Colombia. http://ciencias.bogota. unal.edu.co/menu-principal/publicaciones/bibliotecadigital/flora-de-colombia/ (consulted October, 2021).
Fernández-A., J. L. and M. Hernández-S. 2007. Catálogo de la flora vascular de la cuenca alta del río Subachoque (Cundinamarca, Colombia). Caldasia 29(1): 73-104.

Franco-R., P. and J. Betancur. 1999. La flora del Alto Sumapaz (cordillera Oriental, Colombia). Academia Colombiana de Ciencias Exactas Físicas y Naturales 23: 53-78.

Galindo, R., J. Betancur and J. Cadena. 2003. Estructura y composición florística de cuatro bosques andinos del Santuario de Flora y Fauna Guanentá-alto río Fonce, cordillera Oriental colombiana. Caldasia 25(2): 313-335.

García, N., E. Calderón and G. Galeano. 2005. Frailejones. In: Calderón, E., G. Galeano and N. García (eds.). Libro rojo de plantas de Colombia, Vol. 2: Palmas, frailejones y zamias. Serie libros rojos de especies amenazadas de Colombia, Instituto Alexander von Humboldt - Instituto de Ciencias Naturales de la Universidad Nacional de Colombia Ministerio de Ambiente, Vivienda y Desarrollo Territorial. Bogotá, Colombia. Pp. 225-386.

Gentry, A. 1982. Patterns of neotropical plant species diversity. In: Hechr, M. K., B. Wallace and G. T. Prance (eds.). Evolutionary Biology. Springer. Boston, USA. Pp 1-84

Gentry, A. 1993. A field guide to the families and genera of woody plants of northwest South America (Colombia, Ecuador, Peru) with supplementary notes on herbaceous taxa. Conservation International. Washington, D.C., USA. 895 pp.

Gentry, A. 1995. Patterns of diversity and floristic composition in Neotropical montane forests. In: Churchill, S. P., H. Balslev, E. Forero and J. L. Luteyn (eds.). Biodiversity and Conservation of Neotropical Montane Forest. The New York Botanical Garden. Nueva York, USA. Pp. 103-126.

Gentry, A. 2001. Patrones de diversidad y composición florística en los bosques de las montañas neotropicales. In: Kappelle, M. and A. D. Brown (eds.). Bosques nublados del neotrópico. Instituto Nacional de Biodiversidad. Santo Domingo de Heredia, Costa Rica. Pp. 125-154.

Gil, P. A. 2016. Análisis multitemporal de la vegetación del Macizo de Bijagual, Boyacá. Tunja. Tesis de maestría. Facultad de Ingeniería, Universidad Pedagógica y Tecnológica de Colombia. Tunja, Boyacá, Colombia. 115 pp.

Gil-Leguizamón, P. A., M. E., Morales-Puentes and J. Jácome. 2020. Estructura del bosque altoandino y páramo en el Macizo de Bijagual, Boyacá, Colombia. Revista de Biología 
Tropical 68(3): 765-776. DOI: https://doi.org/10.15517/RBT. V6813.34912

Hernández, A. and N. García. 2006. Las pasifloras. In: García, N. and G. Galeano (eds.). Libro Rojo de Plantas de Colombia, Vol. 3: Las bromelias, las labiadas y las pasifloras. Serie Libros Rojos de Especies Amenazadas de Colombia, Instituto Alexander von Humboldt - Instituto de Ciencias Naturales de la Universidad Nacional de Colombia - Ministerio de Ambiente, Vivienda y Desarrollo Territorial. Bogotá, Colombia. Pp. 583-657.

Idárraga, P. A. and R. Callejas. 2011. Listado de las plantas vasculares del departamento de Antioquia. In: Idárraga, A., R. Ortiz, R. Callejas and M. Merello (eds.). Flora de Antioquia: catálogo de las plantas vasculares, Vol II: Listado de las plantas vasculares del Departamento de Antioquia. Programa expedición Antioquia-2013, Series Biodiversidad y Recursos Naturales, Universidad de Antioquia, Missouri Botanical Garden \& Oficina de Planeación Departamental de la Gobernación de Antioquia, Editorial D’Vinni. Bogotá, Colombia. Pp. 127-922.

IPNI. 2021. International Plant Name Index (IPNI). https://www. ipni.org/ (consulted October, 2021).

IUCN. 2021. IUCN Red List of Threatened Species. International Union for the Conservation of Nature (IUCN). http://www. iucnredlist.org (consulted October, 2021).

Jadán, O., C. Toledo, B. Tepán, H. Cedillo, Á. Peralta, P. Zea, P. Castro and C. Vaca. 2017. Comunidades forestales en bosques secundarios alto-andinos (Azuay, Ecuador). Bosque 38(1): 141-154. DOI: https://doi.org/10.4067/ S0717-92002017000100015

León, J., G. Vélez and A. P. Yepes. 2009. Estructura y composición florística de tres robledales en la región norte de la cordillera Central de Colombia. Revista Biología Tropical 57(4): 11651182. DOI: https://doi.org/10.15517/rbt.v57i4.5454

Marín, C. and J. Betancur. 1997. Estudio florístico en un robledal del Santuario de Flora y Fauna de Iguaque (BoyacáColombia). Academia Colombiana de Ciencias Exactas, Físicas y Naturales 21(80): 249-259.

Medina, W. A., D. C. Macana-García and F. Sánchez. 2015. Aves y mamíferos de bosque altoandino-páramo en el páramo de Rabanal (Boyacá-Colombia). Ciencia en Desarrollo 6(2): 185-198. DOI: https://doi.org/10.19053/01217488.3789
Montenegro, A. L. and O. Vargas. 2008. Atributos vitales de especies leñosas en bordes de bosque altoandino de la Reserva Forestal de Cogua (Colombia). Revista Biología Tropical 56(2): 705-720. DOI: https://doi.org/10.15517/RBT. V56I2.5618

Morales, M., J. Otero, T., van der Hammen, A. Torres, C. Cadena, C. Pedraza, Rodríguez, N., C. Franco, J. C. Betancourth, E. Olaya, E. Posada and L. Cárdenas. 2007. Atlas de páramos de Colombia. Instituto de Investigación de Recursos Biológicos Alexander von Humboldt. Bogotá, Colombia. 208 pp.

Mosquera-Ramos, L. J., D. Robledo-Murillo and A. AsprillaPalacios. 2007. Diversidad florística de dos zonas de bosque tropical húmedo en el municipio de Alto Baudó, ChocóColombia. Acta Biológica Colombiana 12: 75-90.

Olaya-Angarita, J. A., C. N. Díaz-Pérez and M. E. Morales-Puentes. 2019. Composición y estructura de la transición bosquepáramo en el corredor Guantiva-La Rusia (Colombia). Revista de Biología Tropical 67(4): 755-768. DOI: https:// doi.org/10.15517/RBT.V6714.31965

PAC. 2021. Flora de Panama Checklist. Jardín Botánico de Missouri. Saint Louis, USA. http://legacy.tropicos.org/ Project/PAC (consulted October, 2021).

Ramírez-Padilla, B., S. M. Urbano-Apraez and M. S. GonzálezInsuasti. 2015. Flora genérica vascular de la Cuenca alta del Guamués (La Cocha), Nariño, Colombia. Editorial Universitaria - Universidad de Nariño. Pasto, Colombia. 310 pp.

Rangel-Ch., J. O. 1995. Consideraciones sobre la diversidad vegetal de alta montaña en Colombia: Memorias del seminario taller sobre alta montaña colombiana. Academia Colombiana de Ciencias Exactas Físicas y Naturales. Bogotá, Colombia. Pp. 33-60.

Rangel-Ch., J. O. and A. Velásquez. 1997. Métodos de estudio de la vegetación. In: Rangel-Ch., J. O., P. D. Lowy-C. and M. Aguilar-P. (eds.). Colombia diversidad biótica II: Tipos de vegetación en Colombia. Universidad Nacional de Colombia, Facultad de Ciencias, Instituto de Ciencias Naturales, Editorial Unibiblos. Bogotá, Colombia. Pp. 59-87.

Rangel-Ch., J. O., P. D. Lowy-C. and M. Aguilar-P. 1997. La distribución de los tipos de vegetación en las regiones naturales de Colombia, aproximación inicial. In: RangelCh., J. O., P. D. Lowy-C. and M. Aguilar-P (eds.). Colombia 
diversidad biótica II: Tipos de vegetación en Colombia. Universidad Nacional de Colombia, Facultad de Ciencias, Instituto de Ciencias Naturales, Editorial Unibiblos. Bogotá, Colombia. Pp. 383-402.

Rangel-Ch., J. O. 2000. Colombia diversidad biótica III, la región de vida paramuna de Colombia. Universidad Nacional de Colombia, Facultad de Ciencias, Instituto de Ciencias Naturales. Instituto Alexander von Humboldt, Editorial Unibiblos. Bogotá, Colombia. 902 pp.

Segura-Madrigal, M. A., H. J. Andrade-Castañeda and C. A. Mojica-Sánchez. 2019. Estructura, composición florística y almacenamiento de carbono en bosques nativos del páramo de Anaime, Tolima, Colombia. Ciencia Forestal 29(1): 157168. DOI: https://doi.org/10.5902/1980509826551

SFNM. 2021. Schedule of Flora Neotropica Monographs (Phanerogamic Plants). The New York Botanical Garden Press. Bronx, USA. https://www.nybg.org/botany/ofn/ angio.htm (consulted October, 2021).
TPL. 2021. The Plant List, Version 1.1. http://www.theplantlist. org/ (consulted October, 2021).

Tropicos. 2021. Tropicos. Missouri Botanical Garden. Saint Louis, USA. https://www.tropicos.org/home (consulted October, 2021).

Van der Hammen, T. 1998. Plan ambiental de la cuenca alta del río Bogotá: Análisis, problemáticas y soluciones recomendadas. Corporación Autónoma Regional de Cundinamarca(CAR). Bogotá, Colombia. 142 pp.

Van der Hammen, T. and A. M. Cleef. 1983. Datos para la historia de la flora Andina. Revista Chilena de Historia Natural 56: 97-107. 
Appendix: Recorded flora from the high Andean forest of the Bijagual Massif, Boyacá, Colombia. The Eastern cordillera mountains abbreviations: NA: Native Andean, EA: Endemic Andean, End: Endemic, SN San. Marta: Sierra Nevada of Santa Marta, ECO: Endemic Andean from the Eastern cordillera mountains. Departments: Ant: Antioquia, Ara: Arauca, Boy: Boyacá, Cal: Caldas, Cas: Casanare, Cau: Cauca, Ces: Cesar, Cho: Chocó, Cun: Cundinamarca, Gua: Guajira, Hui: Huila, Nor San: Norte de Santander, Mag: Magdalena, Met: Meta, Nar: Nariño, Qui: Quindío, Ris: Risaralda, San: Santander, Tol: Tolima, Val: Valle, Vau: Vaupés. IUCN Red List Status: EN: Endangered, VU: Vulnerable, NT: Near Threatened, LC: Least Concern, NE: Not evaluated. All voucher specimens are deposited in the herbarium UPTC.

\begin{tabular}{|c|c|c|c|}
\hline & $\begin{array}{c}\text { Voucher } \\
\text { P.A. Gil-Leguizamón }\end{array}$ & $\begin{array}{c}\text { Status/Biogeographical } \\
\text { Region }\end{array}$ & $\begin{array}{c}\text { IUCN Red List } \\
\text { Status }\end{array}$ \\
\hline \multicolumn{4}{|l|}{ MAGNOLIIDS } \\
\hline \multicolumn{4}{|l|}{ Chloranthaceae } \\
\hline Hedyosmum colombianum Cuatrec. & $\begin{array}{l}1324,1416,1454,2049 \\
2206,2372,2445,2533\end{array}$ & ECO $(2100-3500 \mathrm{~m})$ & LC \\
\hline Hedyosmum crenatum Occhioni & 1372 & $\begin{array}{l}\text { NA, SN San. Marta (1990- } \\
\qquad 3700 \mathrm{~m})\end{array}$ & NE \\
\hline Hedyosmum parvifolium Cordem. & $1885,2074,2170$ & NA $(2700-3400 \mathrm{~m})$ & NE \\
\hline Hedyosmum translucidum Cuatrec. & $1860,2093,2180$ & NA $(2000-3100 \mathrm{~m})$ & LC \\
\hline \multicolumn{4}{|l|}{ Lauraceae } \\
\hline Nectandra sp. & 2107 & - & - \\
\hline Ocotea cernua (Nees) Mez & 2491 & $\begin{array}{c}\text { NA, SN San. Marta, Valle del } \\
\text { Cauca }(0-1400 \mathrm{~m})\end{array}$ & LC \\
\hline Ocotea sericea Kunth & 1439, 1999 & NA & LC \\
\hline Persea mutisii Kunth & $1551,1626,2072,2274$ & NA $(2000-3500 \mathrm{~m})$ & LC \\
\hline Pleurothyrium velutinum Meisn. & $1646,1804,2112$ & NA $(2250-3900 \mathrm{~m})$ & NE \\
\hline \multicolumn{4}{|l|}{ Monimiaceae } \\
\hline Mollinedia sp. & 2444 & - & - \\
\hline \multicolumn{4}{|l|}{ Piperaceae } \\
\hline Peperomia acuminata Ruiz \& Pav. & $2059,2119,2254$ & NA $(1800-3600 \mathrm{~m})$ & NE \\
\hline Peperomia sp. 1 & $1342,1850,1986$ & - & - \\
\hline Peperomia sp. 2 & 2402 & - & - \\
\hline Piper artanthe C. DC. & $\begin{array}{l}1548,1566,2177,2468 \\
1979\end{array}$ & NA (1600-2950 m) & NE \\
\hline Piper bogotense C. DC. & 1582 & NA $(200-3500 \mathrm{~m})$ & LC \\
\hline Piper irazuanum C. DC. & $\begin{array}{l}\text { 1343, 1386, 1461, 2110, } \\
2392,2443\end{array}$ & NA $(2000-2800 \mathrm{~m})$ & NE \\
\hline \multicolumn{4}{|l|}{ Winteraceae } \\
\hline Drimys granadensis L. $\mathrm{f}$. & $1378,1476,2092,2147$ & NA $(1800-3900 \mathrm{~m})$ & NE \\
\hline \multicolumn{4}{|l|}{ MONOCOTS } \\
\hline \multicolumn{4}{|l|}{ Alstroemeriaceae } \\
\hline Bomarea angustipetala Baker & 1788,1920 & EA (2600-3850 m) & NE \\
\hline Bomarea setacea (Ruiz \& Pav.) Herb. & $\begin{array}{l}1296,1388,1797,1900, \\
2034,2227,2386,2411, \\
1719,1706,2597\end{array}$ & NA (1900-4000m) & LC \\
\hline Bomarea sp. & $1844,2117,2338$ & - & - \\
\hline \multicolumn{4}{|l|}{ Araceae } \\
\hline Anthurium nigrescens Engl. & 1377,2044 & $\begin{array}{l}\text { NA, from Pacífico (150- } \\
\qquad 3600 \mathrm{~m})\end{array}$ & LC \\
\hline Anthurium oxybelium Shott & $\begin{array}{l}\text { 1437, 1957, 2051, 2100, } \\
2201,2244,2354,1414\end{array}$ & NA (1350-3860m) & LC \\
\hline
\end{tabular}


Appendix: Continuation.

\begin{tabular}{|c|c|c|c|}
\hline & $\begin{array}{c}\text { Voucher } \\
\text { P.A. Gil-Leguizamón }\end{array}$ & $\begin{array}{c}\text { Status/Biogeographical } \\
\text { Region }\end{array}$ & $\begin{array}{c}\text { IUCN Red List } \\
\text { Status }\end{array}$ \\
\hline Anthurium sp. 1 & 1875,1382 & - & - \\
\hline Anthurium sp. 2 & 1478 & - & - \\
\hline Anthurium sp. 3 & 1387,1508 & - & - \\
\hline \multicolumn{4}{|l|}{ Asphodelaceae } \\
\hline Eccremis coarctata (Ruiz \& Pav.) Baker & $2191,2503,1828,1905$ & $\begin{array}{l}\text { NA, SN San. Marta, from } \\
\text { Ant, Boy, Cun, Gua, Hui, } \\
\text { Mag, San (1990-3600 m) }\end{array}$ & NE \\
\hline \multicolumn{4}{|l|}{ Bromeliaceae } \\
\hline Greigia stenolepis L.B. Sm. & $\begin{array}{l}\text { 1312, 1381, 1477, 1511, } \\
2403,2451\end{array}$ & ECO $(2500-3720 \mathrm{~m})$ & NT \\
\hline Guzmania gloriosa (André) André ex Mez & $2178,2193,2454$ & NA $(900-3170 \mathrm{~m})$ & LC \\
\hline Guzmania squarrosa (Mez \& Sodiro) L.B. Sm. \& Pittendr. & $1329,1481,2436$ & $\begin{array}{c}\text { NA, Guayana, Macarena } \\
(1000-3680 \mathrm{~m})\end{array}$ & LC \\
\hline Mezobromelia capituligera (Griseb.) J.R. Grant & 2026,2176 & $\begin{array}{l}\text { NA, Amazonía, SN San. } \\
\text { Marta (310-2820 m) }\end{array}$ & LC \\
\hline Puya goudotiana Mez & $\begin{array}{l}\text { 1333, 1904, 2356, 1820, } \\
1408,1771\end{array}$ & ECO $(2760-3550 \mathrm{~m})$ & NT \\
\hline Puya sp. & 2382,2497 & - & - \\
\hline Racinaea riocreuxii (André) M.A. Spencer \& L.B. Sm. & $\begin{array}{l}1562,1618,1633,2007 \\
2069,2135,2296,2522\end{array}$ & NA (1789-3330 m) & LC \\
\hline Racinaea sp. & 2523 & - & - \\
\hline Racinaea tetrantha (Ruiz \& Pav.) M.A. Spencer \& L.B. Sm. & $\begin{array}{l}1617,1893,2158,2521 \\
1680,1681\end{array}$ & $\begin{array}{l}\text { NA, SN San. Marta (1280- } \\
\qquad 3900 \mathrm{~m})\end{array}$ & LC \\
\hline Tillandsia biflora Ruiz \& Pav. & 2306 & $\begin{array}{l}\text { NA, Caribe, SN San. Marta } \\
\qquad(1390-3680 \mathrm{~m})\end{array}$ & LC \\
\hline Tillandsia compacta Griseb. & 1874,2179 & NA (1700-3850 m) & LC \\
\hline Tillandsia complanata Benth. & $1581,1632,2025$ & $\begin{array}{l}\text { NA, SN San. Marta, Valle del } \\
\text { Magdalena (800-3650 m) }\end{array}$ & LC \\
\hline Tillandsia pallescens Betancur \& García Nestor & 2498 & ECO $(2950-3100 \mathrm{~m})$ & NT \\
\hline Tillandsia restrepoana André & 2282 & $\begin{array}{l}\text { NA, SN San. Marta (2300- } \\
2984 \mathrm{~m})\end{array}$ & LC \\
\hline Tillandsia sp. 1 & 2542 & - & - \\
\hline Tillandsia sp. 2 & 1360 & - & - \\
\hline Tillandsia sp. 3 & 2160 & - & - \\
\hline Tillandsia turneri Baker & $1527,1619,2534,1676$ & NA $(2600-3600 \mathrm{~m})$ & LC \\
\hline Vriesea rubrobracteata Rauh & 2311 & ECO $(1000-2850 \mathrm{~m})$ & LC \\
\hline \multicolumn{4}{|l|}{ Cyperaceae } \\
\hline Carex cf. livida (Wahlenb.) Willd. & 1823 & NA (2880-3730 m) & NE \\
\hline Carex jamesonii Boott & 2495 & NA $(1560-4100 \mathrm{~m})$ & LC \\
\hline Carex luridiformis Mack. ex Reiznicek \& S. Gozélez & 2019 & EA $(2500-3800 \mathrm{~m})$ & LC \\
\hline Kyllinga brevifolia Rottb. & 1580 & $\begin{array}{l}\text { NA, Caribe, Orinoquia, } \\
\text { Pacífico, SN San. Marta, } \\
\text { Valle del Cauca (0-2850 m) }\end{array}$ & LC \\
\hline
\end{tabular}


Appendix: Continuation.

\begin{tabular}{|c|c|c|c|}
\hline & $\begin{array}{c}\text { Voucher } \\
\text { P.A. Gil-Leguizamón }\end{array}$ & $\begin{array}{c}\text { Status/Biogeographical } \\
\text { Region }\end{array}$ & $\begin{array}{c}\text { IUCN Red List } \\
\text { Status }\end{array}$ \\
\hline Oreobolus goeppingeri Suess. & 2017,1762 & NA (3000-4200 m) & NE \\
\hline Rhynchospora ruiziana Boeckeler & $1895,2554,2636$ & NA (1200-4000 m) & NE \\
\hline Rhynchospora sp. & $2168,2366,1695,1741$ & - & - \\
\hline \multicolumn{4}{|l|}{ Dioscoreaceae } \\
\hline Dioscorea cf. killipii R. Knuth & 2237 & NA (1700-3680 m) & NE \\
\hline Dioscorea coriacea Humb. \& Bonpl. ex Willd. & $1530,1854,2169$ & $\begin{array}{l}\text { NA, SN San. Marta (1200- } \\
3900 \mathrm{~m})\end{array}$ & NE \\
\hline \multicolumn{4}{|l|}{ Eriocaulaceae } \\
\hline Paepalanthus columbiensis Ruhland & $\begin{array}{l}1554,1903,2085,2363 \\
2557,1694,1733,1777\end{array}$ & EA $(1900-3600 \mathrm{~m})$ & NE \\
\hline Paepalanthus sp. & 2388 & - & - \\
\hline \multicolumn{4}{|l|}{ Juncaceae } \\
\hline Juncus effusus L. & $1573,1639,1821$ & $\begin{array}{l}\text { NA, SN San. Marta (1500- } \\
\qquad 3700 \mathrm{~m})\end{array}$ & LC \\
\hline Luzula gigantea Desv. & 1787 & $\begin{array}{l}\text { NA, SN San. Marta (2700- } \\
4500 \mathrm{~m})\end{array}$ & $\mathrm{NE}$ \\
\hline \multicolumn{4}{|l|}{ Orchidaceae } \\
\hline Anathallis sclerophylla (Lindl.) Pridgeon \& M.W.Chase & 1808,2488 & NA $(1980-3100 \mathrm{~m})$ & $\mathrm{NE}$ \\
\hline Brachionidium tuberculatum Lindl. & 1447,1965 & NA $(280 \mathrm{~m})$ & NE \\
\hline Cranichis sp. & 2389 & - & - \\
\hline Cyrtochilum ramosissimum (Lindl.) Dalström & $\begin{array}{l}1513,2060,2124,2129 \\
1731,2137\end{array}$ & NA $(1900-3600 \mathrm{~m})$ & NE \\
\hline Cyrtochilum sp. & 1987 & - & - \\
\hline Elleanthus cf. ensantus (Lindl.) Rchb. f. & $1374,1628,2225,2346$ & NA $(2300 \mathrm{~m})$ & NE \\
\hline Elleanthus cf. sphaerocephalus Schltr. & $2263,2340,2549$ & NA $(2300 \mathrm{~m})$ & NE \\
\hline Epidendrum sisgaense Hágsater & 1319 & $\mathrm{EA}(2750 \mathrm{~m})$ & NE \\
\hline Epidendrum cf. frutex Rchb. F. & $\begin{array}{l}\text { 1298, 1358, 1475, 2353, } \\
1730,1919\end{array}$ & NA $(2740-3800 \mathrm{~m})$ & NE \\
\hline Epidendrum cf. macrostachyum Lindl. & 1327,2226 & NA, Amazonia (330-2000 m) & NE \\
\hline Epidendrum cylindraceum Lindl. & 1631,2021 & NA $(2700-3200 \mathrm{~m})$ & NE \\
\hline Epidendrum oxysepalum Hágsater \& E. Santiago A. & 1611,1621 & NA $(3200-3950 \mathrm{~m})$ & NE \\
\hline Epidendrum sp. 1 & $1320,1758,2371,2412$ & - & - \\
\hline Epidendrum sp. 2 & 1847 & - & - \\
\hline Epidendrum sp. 3 & 1389,1480 & - & - \\
\hline Epidendrum sp. 4 & $1556,1883,1923,1991$ & - & - \\
\hline Epidendrum sp. 5 & 1501 & - & - \\
\hline Fernandezia tortuosa (Foldats) M.W. Chase & 2334 & NA (3150 m) & NE \\
\hline Fernandezia lanceolata (L.O. Willams) Garay \& Dunst. & $1330,1586,2071,2464$ & NA $(3000-3500 \mathrm{~m})$ & $\mathrm{NE}$ \\
\hline Fernandezia pastii (Rchb.f.) M.W.Chase & $\begin{array}{l}1557,1627,1886,2077 \\
2205,2289,2387\end{array}$ & NA $(2100-4000 \mathrm{~m})$ & NE \\
\hline Fernandezia sp. & 1855 & - & - \\
\hline Hofmeisterella falcata (Linden \& Rchb.f.) Nauray \& A.Galán & 2000 & ECO $(2700 \mathrm{~m})$ & $\mathrm{NE}$ \\
\hline Lepanthes dunstervilleorum Foldats & 2207 & NA (2600-2930 m) & NE \\
\hline
\end{tabular}


Appendix: Continuation.

\begin{tabular}{|c|c|c|c|}
\hline & $\begin{array}{c}\text { Voucher } \\
\text { P.A. Gil-Leguizamón }\end{array}$ & $\begin{array}{c}\text { Status/Biogeographical } \\
\text { Region }\end{array}$ & $\begin{array}{c}\text { IUCN Red List } \\
\text { Status }\end{array}$ \\
\hline Lepanthes sp. 1 & 1492 & - & - \\
\hline Lepanthes sp. 2 & $\begin{array}{l}2061,2094,2198,2256, \\
2342\end{array}$ & - & - \\
\hline Lepanthes sp. 3 & 1558 & - & - \\
\hline Lepanthes sp. 4 & 1326,1426 & - & - \\
\hline Oncidium ornithorhynchum Kunth & $1574,1629,2185$ & NA (2000-3400 m) & NE \\
\hline Pleurothallis cf. secunda Poepp. \& Endl. & 2126 & NA $(2500-3500 \mathrm{~m})$ & $\mathrm{NE}$ \\
\hline Pleurothallis sp. 1 & 1489,2028 & - & - \\
\hline Pleurothallis sp. 2 & 1537,2127 & - & - \\
\hline Pleurothallis sp. 3 & 1803 & - & - \\
\hline Pleurothallis sp. 4 & 2287 & - & - \\
\hline Pleurothallis sp. 5 & 2538 & - & - \\
\hline Stelis cf. aviceps Lindl. & $2195,2484,2541$ & NA $(2000 \mathrm{~m})$ & NE \\
\hline Stelis sp. 1 & 1487 & - & - \\
\hline Stelis sp. 2 & 1806 & - & - \\
\hline Stelis sp. 3 & 2128,2196 & - & - \\
\hline Stelis sp. 4 & 2032 & - & - \\
\hline Stelis sp. 5 & 2218 & - & - \\
\hline Stelis sp. 6 & 2547 & - & - \\
\hline Telipogon sp. & $1609,1873,2293,2332$ & & \\
\hline \multicolumn{4}{|l|}{ Poaceae } \\
\hline Arthrostylidium sp. & 2438 & - & - \\
\hline Aulonemia sp. & $1496,2037,2239$ & - & - \\
\hline Calamagrostis effusa (Kunth) Steud. & $\begin{array}{l}1302,1901,2011,2358, \\
1699,1769,1776,1906, \\
2635\end{array}$ & $\begin{array}{l}\text { NA, SN San. Marta (2500- } \\
\qquad 4500 \mathrm{~m})\end{array}$ & $\mathrm{NE}$ \\
\hline Calamagrostis sp. & 1645 & - & - \\
\hline Chusquea scandens Kunth & $2175,2270,2315$ & NA $(2500-3500 \mathrm{~m})$ & $\mathrm{NE}$ \\
\hline Chusquea sp. 1 & $\begin{array}{l}1314,1349,1357,1876, \\
2133\end{array}$ & - & - \\
\hline Chusquea sp. 2 & $1453,2369,2530$ & - & - \\
\hline Chusquea tessellata Munro & $\begin{array}{l}2016,1712,1940,1397 \\
1698,1707,1734,2596\end{array}$ & NA $(2800-4350 \mathrm{~m})$ & $\mathrm{NE}$ \\
\hline Cortaderia nitida (Kunth) Pilg. & $\begin{array}{l}1303,1553,1825,1944 \\
1402,1740\end{array}$ & NA $(2500-4000 \mathrm{~m})$ & NE \\
\hline Neurolepis aperta (Munro) Pilg. & 1436,1452 & NA $(2500-4000 \mathrm{~m})$ & NE \\
\hline Neurolepis cf. acuminatissima (Munro) Pilg. & 1433,2634 & NA $(2500-4000 \mathrm{~m})$ & $\mathrm{NE}$ \\
\hline \multicolumn{4}{|l|}{ Smilacaceae } \\
\hline Smilax domingensis Willd. & $\begin{array}{l}1805,2075,1869,2105 \\
2216,2248,2271,2285 \\
2510\end{array}$ & $\begin{array}{l}\text { NA, Amazonia, Caribe, } \\
\text { Pacífico, SN San. Marta, } \\
\text { Valle del Cau, Valle del } \\
\text { Magdalena (0-3320 m) }\end{array}$ & $\mathrm{NE}$ \\
\hline Smilax sp. & 1620 & - & - \\
\hline
\end{tabular}


Appendix: Continuation.

\begin{tabular}{|c|c|c|c|}
\hline & $\begin{array}{c}\text { Voucher } \\
\text { P.A. Gil-Leguizamón }\end{array}$ & $\begin{array}{c}\text { Status/Biogeographical } \\
\text { Region }\end{array}$ & $\begin{array}{c}\text { IUCN Red List } \\
\text { Status }\end{array}$ \\
\hline Smilax tomentosa Kunth & 2249,2314 & $\begin{array}{c}\text { NA, Guayana, Macarena } \\
(1700-3680 \mathrm{~m})\end{array}$ & NE \\
\hline \multicolumn{4}{|l|}{ EUDICOTS } \\
\hline \multicolumn{4}{|l|}{ Adoxaceae } \\
\hline Viburnum tinoides L. f. & 1651 & NA $(920-3724 \mathrm{~m})$ & NE \\
\hline Viburnum triphyllum Benth. & $\begin{array}{l}\text { 1483, 1587, 1778, 2182, } \\
2485,2527\end{array}$ & NA (1700-3200 m) & NE \\
\hline \multicolumn{4}{|l|}{ Aquifoliaceae } \\
\hline Ilex obtusata Triana \& Planch. & 1438,1486 & $\begin{array}{l}\text { NA, SN San. Marta (2400- } \\
\qquad 3400 \mathrm{~m})\end{array}$ & LC \\
\hline Ilex pernervata Cuatrec. & 1898,1990 & $\begin{array}{l}\text { NA, SN San. Marta (2000- } \\
\qquad 3600 \mathrm{~m})\end{array}$ & LC \\
\hline \multicolumn{4}{|l|}{ Araliaceae } \\
\hline Hydrocotyle andina Cuatrec. & 1606 & EA (1450-3400 m) & NE \\
\hline Hydrocotyle sphenoloba Wedd. & 1966 & EA (2000-2900 m) & $\mathrm{NE}$ \\
\hline Oreopanax bogotensis Cuatrec. & $1946,2148,2381$ & NA (2050-4117m) & NE \\
\hline Oreopanax sp. & 1522 & - & - \\
\hline Oreopanax incisus (Willd. ex Schult.) Decne. \& Planch. & 2241,2574 & $\begin{array}{l}\text { NA, Orinoquía, from Ant, } \\
\text { Boy, Cal, Cas, Cua, Ces, } \\
\text { Cun, Hui, Qui, Ris, San, Tol } \\
\text { (200-3750 m) }\end{array}$ & NE \\
\hline Oreopanax integrifolius Cuatrec. & 1316,1380 & EA $(2200-2300 \mathrm{~m})$ & LC \\
\hline Oreopanax mutisianus (Kunth) Decne. \& Planch. & 1292 & EA $(2700-3700 \mathrm{~m})$ & LC \\
\hline Schefflera bejucosa Cuatrec. & 2224,2142 & $\begin{array}{c}\text { End, from Ant, Cau, Ris } \\
(1600-3350 \mathrm{~m})\end{array}$ & NE \\
\hline \multicolumn{4}{|l|}{ Asteraceae } \\
\hline Achyrocline satureioides Lam. (DC.) & 2189 & NA (1250-4000m) & NE \\
\hline Ageratina boyacensis R.M. King \& H. Rob. & 1614,1655 & ECO $(2200-3000 \mathrm{~m})$ & NE \\
\hline Ageratina elegans (Kunth) R.M. King \& H. Rob. & $2018,2079,1702$ & NA $(2400-4000 m)$ & NE \\
\hline Ageratina glyptophlebia (B.L. Rob.) R.M. King \& H. Rob. & 2164 & NA (1850-4050m) & NE \\
\hline Ageratina pseudochilca (Benth.) R.M. King \& H. Rob. & 2228,2145 & NA $(2600-3500 m)$ & NE \\
\hline Ageratina sp. 1 & $1896,2465,2628$ & - & - \\
\hline Ageratina sp. 2 & 2481 & - & - \\
\hline Ageratina sp. 3 & 2529 & - & - \\
\hline Ageratina tinifolia (Kunth) R.M. King \& H. Rob. & $\begin{array}{l}1427,1499,1948,2009 \\
1723,1727,1934,2614, \\
1404,1755\end{array}$ & NA (1350-3900m) & NE \\
\hline Alloispermum pachensis (Hieron.) H. Rob. & 1578 & NA (1500-3100m) & NE \\
\hline Alloispermum sp. & 2471 & - & - \\
\hline Baccharis brachylaenoides DC. & 2230 & NA (500-3550m) & LC \\
\hline Baccharis latifolia (Ruiz \& Pav.) Pers. & 1634 & $\begin{array}{l}\text { NA, Valle from Magdalena } \\
\qquad(1400-4000 \mathrm{~m})\end{array}$ & LC \\
\hline Critoniopsis sp. 1 & 1295,2661 & $\begin{array}{l}\text { End. from Boy (2000- } \\
\qquad 3500 \mathrm{~m})\end{array}$ & - \\
\hline
\end{tabular}


Appendix: Continuation.

\begin{tabular}{|c|c|c|c|}
\hline & $\begin{array}{c}\text { Voucher } \\
\text { P.A. Gil-Leguizamón }\end{array}$ & $\begin{array}{c}\text { Status/Biogeographical } \\
\text { Region }\end{array}$ & $\begin{array}{c}\text { IUCN Red List } \\
\text { Status }\end{array}$ \\
\hline Critoniopsis sp. 2 & 2279 & - & - \\
\hline Diplostephium cf. bicolor S.F. Blake & 1390,1605 & NA $(2500-3900 m)$ & NE \\
\hline Diplostephium floribundum (Benth.) Wedd. & 2322,1749 & EA (2580-4000 m) & NE \\
\hline Diplostephium huertasii Cuatrec. & 2238 & ECO $(2850-3450 m)$ & NE \\
\hline Diplostephium juajibioyi Cuatrec. & 1311,1519 & ECO (3530-3900m) & NE \\
\hline Diplostephium oblongifolium Cuatrec. & 2286,2322 & ECO from Nor San $(2770 \mathrm{~m})$ & EN \\
\hline Diplostephium rosmarinifolium (Benth.) Wedd. & $2291,2308,2505$ & NA $(2000-3900 m)$ & NE \\
\hline Diplostephium tenuifolium Cuatrec. & $1871,2479,2566$ & ECO $(2200-3900 \mathrm{~m})$ & LC \\
\hline Espeletia boyacensis Cuatrec. & $\begin{array}{l}2571,1822,1917,2347 \\
2577\end{array}$ & ECO $(2345-3900 \mathrm{~m})$ & LC \\
\hline Espeletia murilloi Cuatrec. & $\begin{array}{l}1902,1409,1696,1732, \\
1764,2602,2348,2666, \\
2671\end{array}$ & ECO $(2700-3700 \mathrm{~m})$ & LC \\
\hline Gynoxys sp. & 2480 & - & - \\
\hline Jungia coarctata Hieron & 1514 & NA $(2100-3600 m)$ & NE \\
\hline Jungia sp. & 2418 & - & - \\
\hline Mikania aristei B.L. Rob. & $\begin{array}{l}1376,1441,1616,1836, \\
2033,2122,2197,2250, \\
2379,2448\end{array}$ & NA $(1760-3500 m)$ & NE \\
\hline Mikania cf. szyszylowiczii Hieron & 2156,1535 & NA (1800-3450m) & NE \\
\hline Mikania sp. 1 & 2499, 2519 & - & - \\
\hline Mikania sp. 2 & 2507 & - & - \\
\hline Munnozia senecionidis Benth. & $\begin{array}{l}1542,1794,1837,2043 \\
2235,2310,2367,2435 \\
2539\end{array}$ & NA $(950-3820 m)$ & NE \\
\hline Oritrophium peruvianum (Lam.) Cuatrec. & 2668 & $\begin{array}{c}\text { NA, from Ant, Ara, Cal, Boy, } \\
\text { Cau, Ces, Cho, Cun, Gua, } \\
\text { Hui, Mag, Met, Nar, Nor } \\
\text { San, Qui, Ris, San, Tol, Val } \\
(2700-4630 \text { m) }\end{array}$ & NE \\
\hline Pentacalia cf. tolimensis (Sch. Bip. ex Wedd.) Cuatrec. & $1283,2612,2647,2410$ & NA (1750-4300 m) & NE \\
\hline Pentacalia corymbosa (Benth.) Cuatrec. & 2502,2023 & ECO $(1800-4000 \mathrm{~m})$ & NE \\
\hline Pentacalia kleinioides (Kunth) Cuatrec. & 2159 & NA (1900-3500 m) & NE \\
\hline Pentacalia pulchella (Kunth) Cuatrec. & $\begin{array}{l}1307,1608,1880,1968, \\
2161,2281,2323,2508, \\
1922\end{array}$ & NA $(1900-3850 \mathrm{~m})$ & NE \\
\hline Pentacalia sp. 1 & 1861 & - & - \\
\hline Pentacalia sp. 2 & 2385 & - & - \\
\hline Pentacalia sp. 3 & 2467 & - & - \\
\hline Pentacalia sp. 4 & 2537 & - & - \\
\hline Pentacalia theifolia (Benth.) Cuatrec. & $1528,1969,2166$ & NA (1800-3500 m) & NE \\
\hline Pentacalia trianae (Klatt) Cuatrec. & 2005 & NA $(1200-3950 \mathrm{~m})$ & NE \\
\hline Pentacalia trichopus (Benth.) Cuatrec. & $1395,1421,1423,1407$ & NA $(2800-3930 \mathrm{~m})$ & LC \\
\hline Pentacalia vaccinioides (Kunth) Cuatrec. & 1308 & NA (3000-3990 m) & NE \\
\hline
\end{tabular}


Appendix: Continuation.

\begin{tabular}{|c|c|c|c|}
\hline & $\begin{array}{c}\text { Voucher } \\
\text { P.A. Gil-Leguizamón }\end{array}$ & $\begin{array}{c}\text { Status/Biogeographical } \\
\text { Region }\end{array}$ & $\begin{array}{c}\text { IUCN Red List } \\
\text { Status }\end{array}$ \\
\hline Verbesina cf. pennellii S.F. Blake & 2626 & $\begin{array}{l}\text { End, from Cho, Cund, Hui, } \\
\text { Qui, Tol, Val (1300-2600 m) }\end{array}$ & NE \\
\hline Verbesina crassiramea S.F. Blake & 1635 & ECO $(2000-3510 \mathrm{~m})$ & NE \\
\hline \multicolumn{4}{|l|}{ Begoniaceae } \\
\hline Begonia cornuta L. B. Sm. \& B. G. Schub. & 1867,2407 & NA $(2200-3450 \mathrm{~m})$ & NE \\
\hline Begonia ferruginea L. f. & $\begin{array}{l}1488,1529,1834,2045 \\
2429,2431,1935,2143\end{array}$ & NA $(1000-4000 \mathrm{~m})$ & NE \\
\hline Begonia urticae L. f. & $\begin{array}{l}\text { 1289, 1364, 1468, 1517, } \\
1779,1882,2027\end{array}$ & NA $(1600-4000 \mathrm{~m})$ & LC \\
\hline \multicolumn{4}{|l|}{ Berberidaceae } \\
\hline Berberis muiscarum L.A. Camargo & $1284,1336,1653$ & EA $(2780-3640 \mathrm{~m})$ & NE \\
\hline Berberis sp. & 2010 & - & - \\
\hline \multicolumn{4}{|l|}{ Boraginaceae } \\
\hline Varronia cylindrostachya Ruiz R Pav. & 1644 & NA $(520-3400 \mathrm{~m})$ & LC \\
\hline \multicolumn{4}{|l|}{ Brunelliaceae } \\
\hline Brunellia cf. comocladifolia Bonpl. & $\begin{array}{l}1442,1465,1800,1835 \\
2035,2104,2325,2551\end{array}$ & $\begin{array}{l}\text { NA, SN San. Marta (800- } \\
3100 \mathrm{~m})\end{array}$ & LC \\
\hline Brunellia propinqua Kunth & 2393,$2447 ; 1515,2223$ & ECO $(1800-3850 \mathrm{~m})$ & LC \\
\hline \multicolumn{4}{|l|}{ Campanulaceae } \\
\hline Burmeistera globosa E. Wimm & $1384,2351,2419$ & ECO $(2400-3400 \mathrm{~m})$ & NE \\
\hline Centropogon sp. & $1571,1600,2428$ & - & - \\
\hline Siphocampylus retrorsus Vatke \& E. Wimm. & $\begin{array}{l}1345,1385,1435,1518 \\
1523,2121,2208,2288 \\
2380,2550,1924,1937\end{array}$ & ECO $(1000-3500 \mathrm{~m})$ & NE \\
\hline Siphocampylus sp. & 2192,2493 & - & - \\
\hline \multicolumn{4}{|l|}{ Caprifoliaceae } \\
\hline Valeriana clematitis Kunth. & 1309,1352 & - & NE \\
\hline \multicolumn{4}{|l|}{ Caryophyllaceae } \\
\hline Stellaria cuspidata D.F.K. Schltdl. & 1959,2442 & NA $(820-3820 \mathrm{~m})$ & $\mathrm{NE}$ \\
\hline \multicolumn{4}{|l|}{ Celastraceae } \\
\hline Maytenus prunifolia C. Presl. & 2470 & NA $(2290-3600 \mathrm{~m})$ & NE \\
\hline \multicolumn{4}{|l|}{ Clethraceae } \\
\hline Clethra fagifolia (Kunth) Sleumer & 2257,2261 & $\begin{array}{l}\text { NA, SN San. Marta (1500- } \\
3420 \mathrm{~m})\end{array}$ & NE \\
\hline \multicolumn{4}{|l|}{ Clusiaceae } \\
\hline Clusia alata Planch. \& Triana & $\begin{array}{l}2365,1322,1356,1420 \\
1482,1657\end{array}$ & EA $(1100-3500 \mathrm{~m})$ & LC \\
\hline Clusia elliptica Kunth & $\begin{array}{l}1331,1379,1383,1429, \\
1455,1505,1533,1599, \\
1832,1972,2046,2087, \\
2446,2490,2515,2024\end{array}$ & $\mathrm{EA}(2550-3600 \mathrm{~m})$ & LC \\
\hline Clusia multiflora Kunth & $1868,2047,2514$ & $\begin{array}{c}\text { NA, Guayana, Macarena, } \\
\text { Pacífico, SN San. Marta } \\
(80-3500 \mathrm{~m})\end{array}$ & LC \\
\hline
\end{tabular}


Appendix: Continuation.

\begin{tabular}{|c|c|c|c|}
\hline & $\begin{array}{c}\text { Voucher } \\
\text { P.A. Gil-Leguizamón }\end{array}$ & $\begin{array}{c}\text { Status/Biogeographical } \\
\text { Region }\end{array}$ & $\begin{array}{c}\text { IUCN Red List } \\
\text { Status }\end{array}$ \\
\hline \multicolumn{4}{|l|}{ Cunoniaceae } \\
\hline Weinmannia balbisiana Kunth & $\begin{array}{l}1538,1842,2042,2095 \\
2262,2423,2517\end{array}$ & NA $(710-3050 \mathrm{~m})$ & LC \\
\hline Weinmannia fagaroides Kunth & $\begin{array}{l}1559,1597,2307,2520, \\
1812,1410,1916\end{array}$ & $\begin{array}{l}\text { NA, SN San. Marta (2550- } \\
\qquad 4000 \mathrm{~m})\end{array}$ & LC \\
\hline Weinmannia reticulata Ruiz \& Pav. & $\begin{array}{l}1630,1652,1988,2098 \\
2427,2545,1931,1674\end{array}$ & NA $(1800-3440 \mathrm{~m})$ & LC \\
\hline Weinmannia rollottii Killip & $\begin{array}{l}1287,1368,1451,1495, \\
1843,2260,2276,2364, \\
2424,1411,1690\end{array}$ & NA $(2250-3450 \mathrm{~m})$ & LC \\
\hline Weinmannia tomentosa $\mathrm{L} . \mathrm{f}$. & $\begin{array}{l}1332,2231,2269,2309, \\
1726,1939\end{array}$ & NA (1700-3724 m) & LC \\
\hline \multicolumn{4}{|l|}{ Elaeocarpaceae } \\
\hline Vallea stipularis L. f. & 1563,1648 & $\begin{array}{l}\text { NA, SN San. Marta (1990- } \\
\qquad 4300 \mathrm{~m})\end{array}$ & LC \\
\hline \multicolumn{4}{|l|}{ Ericaceae } \\
\hline Bejaria resinosa Mutis ex. L. f. & $2558,1713,1818,1685$ & NA (1750-3900 m) & LC \\
\hline Cavendishia bracteata (Ruiz \& Pav. ex J.St. Hil.) Hoerold & $2229,2290,2437$ & $\begin{array}{l}\text { NA, SN San. Marta (1000- } \\
3820 \mathrm{~m})\end{array}$ & LC \\
\hline Disterigma alaternoides (Kunth) Nied. & $\begin{array}{l}1286,1369,1857,2116 \\
2264,2416,1938,1739\end{array}$ & NA $(1500-3600 \mathrm{~m})$ & LC \\
\hline Disterigma cryptocalyx A.C. Sm. & 1462,2508 & NA $(1000-2750 \mathrm{~m})$ & $\mathrm{NE}$ \\
\hline Disterigma empetrifolium (Kunth) Drude & $\begin{array}{l}1285,1503,2361,2567, \\
1928,1686,1745,1768, \\
2641\end{array}$ & NA $(2500-4500 \mathrm{~m})$ & LC \\
\hline Disterigma sp. 1 & $1347,1594,1784$ & - & - \\
\hline Disterigma sp. 2 & 1493 & - & - \\
\hline Gaultheria anastomosans (L. f.) Kunth & $\begin{array}{l}1305,1560,1971,2076, \\
2082,2236,2494,2565, \\
1721,1932,1943,2607, \\
1688,1738\end{array}$ & $\begin{array}{l}\text { NA, SN San. Marta (2500- } \\
\qquad 4100 \mathrm{~m})\end{array}$ & NE \\
\hline Gaultheria buxifolia Willd. & 1950 & $\begin{array}{l}\text { NA, SN San. Marta (2000- } \\
\qquad 3200 \mathrm{~m})\end{array}$ & NE \\
\hline Gaultheria erecta Vent. & $\begin{array}{l}1301,1889,2187,2301, \\
2343,2360,1941,1406, \\
1767\end{array}$ & $\begin{array}{l}\text { NA, SN San. Marta (2000- } \\
\qquad 3700 \mathrm{~m})\end{array}$ & LC \\
\hline Gaultheria rigida Kunth & $1498,2478,2568,2639$ & NA $(2700-3500 \mathrm{~m})$ & NE \\
\hline Gaultheria strigosa Benth. & $\begin{array}{l}\text { 1362, 1425, 1506, 1792, } \\
2379,2461\end{array}$ & NA $(2400-3950 \mathrm{~m})$ & $\mathrm{NE}$ \\
\hline Macleania rupestris (Kunth) A.C. Sm. & $\begin{array}{l}1288,1463,1464,1549, \\
1790,1856,2063,2268, \\
2355,2368,2466,1816, \\
1929,1700\end{array}$ & $\begin{array}{l}\text { NA, SN San. Marta (2000- } \\
\qquad 4100 \mathrm{~m})\end{array}$ & LC \\
\hline
\end{tabular}


Appendix: Continuation.

\begin{tabular}{|c|c|c|c|}
\hline & $\begin{array}{c}\text { Voucher } \\
\text { P.A. Gil-Leguizamón }\end{array}$ & $\begin{array}{c}\text { Status/Biogeographical } \\
\text { Region }\end{array}$ & $\begin{array}{c}\text { IUCN Red List } \\
\text { Status }\end{array}$ \\
\hline Pernettya prostrata (Cav.) DC. & $\begin{array}{l}\text { 1294, 1335, 1810, 2609, } \\
1401,1912\end{array}$ & $\begin{array}{l}\text { NA, SN San. Marta (2000- } \\
\qquad 4525 \mathrm{~m})\end{array}$ & LC \\
\hline Plutarchia guascensis (Cuatrec.) A.C. Sm. & 1664 & $\begin{array}{l}\text { End. from Cun, Boy, San } \\
(2800-4180 \mathrm{~m})\end{array}$ & VU \\
\hline Psammisia cf. idalima A.C. Sm. & 1471 & NA (2000-2500 m) & NE \\
\hline Sphyrospermum buxifolium Poepp. \& Endl. & 1796 & $\begin{array}{l}\text { NA, SN San. Marta (1200- } \\
\qquad 3200 \mathrm{~m})\end{array}$ & NE \\
\hline Sphyrospermum cordifolium Benth. & $1952,2054,2258,2303$ & $\begin{array}{l}\text { NA, Pacífico, SN San. Marta } \\
(100-3600 \mathrm{~m})\end{array}$ & NE \\
\hline Sphyrospermum sp. 1 & 1592 & - & - \\
\hline Sphyrospermum sp. 2 & 2151 & - & - \\
\hline Thibaudia floribunda Kunth & 1543 & NA, Pacífico (200-4180 m) & LC \\
\hline \multicolumn{4}{|l|}{ Escalloniaceae } \\
\hline Escallonia myrtilloides L. f. & $\begin{array}{l}1334,1363,1424,1785 \\
1914,1980\end{array}$ & $\begin{array}{l}\text { NA, SN San. Marta (2500- } \\
3900 \mathrm{~m})\end{array}$ & NE \\
\hline \multicolumn{4}{|l|}{ Fabaceae } \\
\hline Lupinus bogotensis Benth. & 2349 & $\begin{array}{c}\text { NA, from Ant, Boy, Cal, Cun, } \\
\text { Met, Nor San (1700-3700 } \\
\text { m) }\end{array}$ & LC \\
\hline \multicolumn{4}{|l|}{ Fagaceae } \\
\hline Quercus humboldtii Bonpl. & 2277 & $\begin{array}{l}\text { Native and cultivated } \\
\text { Andean }(1400-3300 \mathrm{~m})\end{array}$ & VU \\
\hline \multicolumn{4}{|l|}{ Gentianaceae } \\
\hline Halenia asclepiadea (Kunth) G. Don & 1976, 1746 & EA $(2700-4000 \mathrm{~m})$ & NE \\
\hline Macrocarpaea macrophylla (Kunth) Gilg & $2084,1872,2489,2553$ & NA (1250-3200 m) & LC \\
\hline Symbolanthus calygonus (Ruiz \& Pav.) Griseb. ex Gilg & 2083,2113 & $\begin{array}{c}\text { NA, from Ant, Bol, Boy, Cun, } \\
\text { Met, Nor San, San, Tol } \\
(1050-3300 \mathrm{~m})\end{array}$ & NE \\
\hline \multicolumn{4}{|l|}{ Geraniaceae } \\
\hline Geranium cf. alonsoi Aedo & 1313,1911 & ECO $(2900-3700 \mathrm{~m})$ & NE \\
\hline \multicolumn{4}{|l|}{ Gesneriaceae } \\
\hline Columnea strigosa Benth. & $\begin{array}{l}1484,1526,1598,1881 \\
1953,2052,2215,2243 \\
2341,2344,2373,2417 \\
2345\end{array}$ & NA $(1100-4350 \mathrm{~m})$ & NE \\
\hline Glossoloma ichthyoderma (Hanst.) J.L. Clark & 1862,2118 & NA $(1000-3900 m)$ & NE \\
\hline \multicolumn{4}{|l|}{ Gunneraceae } \\
\hline Gunnera schultesii L.E. Mora & 1567,1949 & ECO $(2840-3350 \mathrm{~m})$ & NE \\
\hline \multicolumn{4}{|l|}{ Hypericaceae } \\
\hline Hypericum laricifolium Juss. & 1774, 1908 & $\begin{array}{l}\text { NA, SN San. Marta (2500- } \\
\qquad 4300 \mathrm{~m})\end{array}$ & LC \\
\hline Hypericum lycopodioides Triana \& Planch. & $\begin{array}{l}1297,1892,2162,2357 \\
2564 \text { о } 2554,1811,1925 \\
1400,1703\end{array}$ & ECO $(2850-4100 \mathrm{~m})$ & NE \\
\hline
\end{tabular}


Appendix: Continuation.

\begin{tabular}{|c|c|c|c|}
\hline & $\begin{array}{c}\text { Voucher } \\
\text { P.A. Gil-Leguizamón }\end{array}$ & $\begin{array}{c}\text { Status/Biogeographical } \\
\text { Region }\end{array}$ & $\begin{array}{c}\text { IUCN Red List } \\
\text { Status }\end{array}$ \\
\hline Hypericum sp. & 1602 & - & - \\
\hline \multicolumn{4}{|l|}{ Lamiaceae } \\
\hline Lepechinia conferta (Benth.) Epling & 2190, 1978 & $\begin{array}{l}\text { NA, SN San. Marta (2300- } \\
3350 \mathrm{~m})\end{array}$ & LC \\
\hline \multicolumn{4}{|l|}{ Loranthaceae } \\
\hline Gaiadendron punctatum (Ruíz \& Pav.) G. Don & $\begin{array}{l}1610,2188,2240,2483, \\
2544,2020\end{array}$ & $\begin{array}{l}\text { NA, SN San. Marta (1330- } \\
3950 \mathrm{~m})\end{array}$ & LC \\
\hline \multicolumn{4}{|l|}{ Melastomataceae } \\
\hline Axinaea scutigera Triana & 2090 & NA $(1100-2970 \mathrm{~m})$ & LC \\
\hline Bucquetia glutinosa (L. f.) DC. & $\begin{array}{l}\text { 1304, 1596, 2572, 1722, } \\
1396\end{array}$ & EA $(2100-4104 \mathrm{~m})$ & LC \\
\hline Meriania brachycera (Naudin) Mend. \& Fern. Alonso & 2036, 2089, 2219, 2221 & NA $(2000-3350 \mathrm{~m})$ & NE \\
\hline Miconia cataractae Triana & $\begin{array}{l}1328,1359,1516,1852, \\
1899,1992,2065,2108, \\
2165,2321,2326,2525, \\
1760\end{array}$ & NA $(2500-3900 \mathrm{~m})$ & $\mathrm{NE}$ \\
\hline Miconia cf. chionophila Naudin & 1370 & NA (2880-4250 m) & NE \\
\hline Miconia cladonia Gleason & 1422,1791 & NA (2000-3300 m) & NE \\
\hline Miconia cleefii L. Uribe & 1299 & ECO $(3300-3900 \mathrm{~m})$ & LC \\
\hline Miconia cundinamarcensis Wurdack & $\begin{array}{l}1325,1415,1445,1448, \\
1591,1624,1870,1888, \\
1993,2132,2154,2352, \\
2406,2524\end{array}$ & EA $(960-3300 \mathrm{~m})$ & LC \\
\hline Miconia denticulata Naudin & 2130 & NA $(2050-3400 \mathrm{~m})$ & NE \\
\hline Miconia dolichopoda Naudin & $\begin{array}{l}1434,1845,1960,1981 \\
2149\end{array}$ & $\begin{array}{l}\text { NA, SN San. Marta (1600- } \\
\qquad 2900 \mathrm{~m})\end{array}$ & LC \\
\hline Miconia jahnii Pittier & $1572,1636,2397$ & NA (1100-3700 m) & NE \\
\hline Miconia ligustrina (Sm.) Triana & $\begin{array}{l}1428,1512,1603,1604, \\
1947,2008,2091,2184, \\
1936,1753\end{array}$ & $\begin{array}{l}\text { NA, SN San. Marta (2050- } \\
\qquad 3800 \mathrm{~m})\end{array}$ & NE \\
\hline Miconia myrtillifolia Naudin & 2171,2500 & NA $(2000-3600 \mathrm{~m})$ & $\mathrm{NE}$ \\
\hline Miconia plethorica Naudin & $1858,2384,2449$ & EA $(2150-3000 \mathrm{~m})$ & LC \\
\hline Miconia sp. & $1472,1568,2604$ & - & - \\
\hline Miconia squamulosa Triana & 2469 & NA $(2100-3600 \mathrm{~m})$ & NE \\
\hline Miconia stipularis Naudin & $\begin{array}{l}\text { 1444, 1457, 1534, 1841, } \\
1884,2055,2543\end{array}$ & EA $(1320-3400 \mathrm{~m})$ & LC \\
\hline Miconia theizans (Bonpl.) Cogn. & $\begin{array}{l}2404,2441 ; 1590,2401, \\
1678\end{array}$ & $\begin{array}{l}\text { NA, Guayana, Macarena, } \\
\text { Pacífico, SN San. Marta, } \\
\text { Valle del Cuaca, Valle del } \\
\text { Magdalena (230-3900 m) }\end{array}$ & $\mathrm{NE}$ \\
\hline Monochaetum meridense Naudin & 2330,2336 & NA $(1250-3275 \mathrm{~m})$ & $\mathrm{NE}$ \\
\hline Monochaetum myrtoideum Naudin & $1638,2167,2569,1814$ & NA (1900-3900 m) & $\mathrm{NE}$ \\
\hline Monochaetum uribei Wurdack & 2203,2272 & ECO $(2300-3200 \mathrm{~m})$ & $\mathrm{NE}$ \\
\hline
\end{tabular}


Appendix: Continuation.

\begin{tabular}{|c|c|c|c|}
\hline & $\begin{array}{c}\text { Voucher } \\
\text { P.A. Gil-Leguizamón }\end{array}$ & $\begin{array}{c}\text { Status/Biogeographical } \\
\text { Region }\end{array}$ & $\begin{array}{c}\text { IUCN Red List } \\
\text { Status }\end{array}$ \\
\hline Tibouchina grossa (L. f.) Cogn. & $\begin{array}{l}1393,1494,1793,2434, \\
1412,1693\end{array}$ & NA $(1850-4500 \mathrm{~m})$ & NE \\
\hline \multicolumn{4}{|l|}{ Myricaceae } \\
\hline Morella funckii (Chev.) Parra-Os. & 1656,2492 & $\begin{array}{l}\text { NA, SN San. Marta (1990- } \\
\qquad 3700 \mathrm{~m})\end{array}$ & $\mathrm{NE}$ \\
\hline Morella parvifolia (Benth.) Parra-Os. & 1521,2284 & NA $(1600-3800 \mathrm{~m})$ & NE \\
\hline \multicolumn{4}{|l|}{ Primulaceae } \\
\hline Cybianthus iteoides (Benth.) G.Agostini & $\begin{array}{l}1443,1466,1532,1555, \\
1588,1853,2213,2251, \\
2253,2305,2374\end{array}$ & NA $(1200-3725 \mathrm{~m})$ & NE \\
\hline Geissanthus andinus Mez & $\begin{array}{l}1509,1525,1584,1998, \\
2155,2370,2540,1725, \\
1933,1689,1945,2146\end{array}$ & NA $(1825-3700 \mathrm{~m})$ & NE \\
\hline Geissanthus quindiensis Mez & $\begin{array}{l}\text { 1789, 1833, 1848, 2048, } \\
2153,2487\end{array}$ & NA (2600-3700 m) & NE \\
\hline Myrsine coriacea (Sw.) R. Br. ex Roem. \& Schult. & 1575,2475 & $\begin{array}{l}\text { NA, Caribe, Pacífico \& Valle } \\
\text { del Magdalena (820-3360 } \\
\text { m) }\end{array}$ & $\mathrm{NE}$ \\
\hline Myrsine dependens (Ruiz \& Pav.) Spreng. & 1351 & $\begin{array}{l}\text { NA, SN San. Marta (2500- } \\
3800 \mathrm{~m})\end{array}$ & LC \\
\hline \multicolumn{4}{|l|}{ Myrtaceae } \\
\hline Myrcianthes rhopaloides (Kunth) McVaugh & $1637,1649,2006,1569$ & NA (1800-3200) & LC \\
\hline Myrcianthes sp. & 2463 & - & - \\
\hline Ugni myricoides (Kunth) O. Berg & 1897,2561 & NA (2190-3500 m) & LC \\
\hline \multicolumn{4}{|l|}{ Onagraceae } \\
\hline Fuchsia canescens Benth. & $1951,2610,1759$ & EA $(2800-3400 \mathrm{~m})$ & NE \\
\hline \multicolumn{4}{|l|}{ Oxalidaceae } \\
\hline Oxalis medicaginea Kunth & 1391, 1799, 1955 & NA $(1400-4000 \mathrm{~m})$ & LC \\
\hline Oxalis sp. 1 & 1306 & - & - \\
\hline Oxalis sp. 2 & 1849,2432 & - & - \\
\hline
\end{tabular}

\section{Passifloraceae}

Passiflora adulterina L. f.

Passiflora bogotensis Benth.

Passiflora sp. 1

Passiflora sp. 2

\section{Pentaphylacaceae}

Freziera bonplandiana Tul.

Ternstroemia cf. camelliifolia Linden \& Planch.
1682

2140

1846, 2031

2452
End. from Boy, Cun, Qui

$$
\text { (2600-3500 m) }
$$

LC

NA, SN San. Marta, en Boy,

Cal, Cun, Gua, Mag, San

(2000-3000 m)
1967,2405

$2067,2202,2234,2266$,

2556,2144

2252
NA, SN San. Marta (2500-

$$
3700 \mathrm{~m} \text { ) }
$$

$\mathrm{NE}$

NA (2800-3300 m)

NE 
Appendix: Continuation.

\begin{tabular}{|c|c|c|c|}
\hline & $\begin{array}{c}\text { Voucher } \\
\text { P.A. Gil-Leguizamón }\end{array}$ & $\begin{array}{c}\text { Status/Biogeographical } \\
\text { Region }\end{array}$ & $\begin{array}{c}\text { IUCN Red List } \\
\text { Status }\end{array}$ \\
\hline \multicolumn{4}{|l|}{ Phyllanthaceae } \\
\hline Hieronyma rufa P. Franco & $2109,2297,2320,2350$ & $\begin{array}{c}\text { EA, from Ant, Boy, Cun, Cau, } \\
\text { Vau }(2300-3350 \mathrm{~m})\end{array}$ & NT \\
\hline \multicolumn{4}{|l|}{ Phytolaccaceae } \\
\hline Phytolacca bogotensis Kunth & 1961 & NA (1560-3600 m) & LC \\
\hline \multicolumn{4}{|l|}{ Plantaginaceae } \\
\hline Sibthorpia repens (L.) Kuntze & 1354,1956 & NA $(3100-3900 \mathrm{~m})$ & NE \\
\hline \multicolumn{4}{|l|}{ Polygalaceae } \\
\hline Monnina bracteata Chodat & $1440,2246,2247$ & EA (1400-2400 m) & $\mathrm{NE}$ \\
\hline Monnina salicifolia Ruiz \& Pav. & $1650,2012,2509$ & $\begin{array}{l}\text { NA, SN San. Marta (1900- } \\
\qquad 4150 \mathrm{~m})\end{array}$ & NE \\
\hline \multicolumn{4}{|l|}{ Polygonaceae } \\
\hline Muehlenbeckia tamnifolia (Kunth) Meisn. & $1392,1550,2004,1724$ & $\begin{array}{l}\text { NA, Valle del Magdalena } \\
\qquad(390-3900 \mathrm{~m})\end{array}$ & NE \\
\hline Polygonum nepalense Meisn. & 1675 & Nativ Asia, from Ant, Hui & NE \\
\hline \multicolumn{4}{|l|}{ Rhamnaceae } \\
\hline Frangula goudotiana (Triana \& Planch.) Grubov & 2194 & NA $(2000-3700 \mathrm{~m})$ & NE \\
\hline \multicolumn{4}{|l|}{ Rosaceae } \\
\hline Hesperomeles obtusifolia (Pers.) Lindl. & $\begin{array}{l}1350,2078,2298,2339 \\
1729,1974,2482,2562\end{array}$ & NA $(2200-3800 \mathrm{~m})$ & LC \\
\hline Lachemilla pectinata (Kunth) Rothm. & 1642 & NA (1800-3400 m) & NE \\
\hline Prunus opaca (Benth.) Walp. & 2299 & NA $(1860-3300 \mathrm{~m})$ & LC \\
\hline Rubus compactus Benth & 1601 & NA $(2500-3600 \mathrm{~m})$ & $\mathrm{NE}$ \\
\hline Rubus coriaceus Poir. & 2377 & NA $(2800-4000 \mathrm{~m})$ & NE \\
\hline Rubus gachetensis A. Berger & 2013 & EA $(2300-3100 \mathrm{~m})$ & NE \\
\hline Rubus guyanensis Focke & 1640 & NA $(2000-3000 \mathrm{~m})$ & NE \\
\hline Rubus nubigenus Kunth & 1531,1851 & $\begin{array}{l}\text { NA, SN San. Marta (2500- } \\
3600 \mathrm{~m})\end{array}$ & NE \\
\hline \multicolumn{4}{|l|}{ Rubiaceae } \\
\hline Arcythophyllum muticum (Wedd.) Standl. & 2014 & $\begin{array}{l}\text { NA, SN San. Marta (1700- } \\
\qquad 4400 \mathrm{~m})\end{array}$ & $\mathrm{NE}$ \\
\hline Arcythophyllum nitidum (Kunth) Schltdl. & $\begin{array}{l}1552,1894,2233,2275 \\
2474,2506,2559,1815 \\
1930,1413,1687,1744\end{array}$ & $\begin{array}{l}\text { NA, SN San. Marta (630- } \\
4500 \mathrm{~m})\end{array}$ & $\mathrm{NE}$ \\
\hline Galianthe cf. bogotensis (Kunth) E.L.Cabral \& Bacigalupo & 1579 & EA $(2200-3575 \mathrm{~m})$ & NE \\
\hline Galium hypocarpium (L.) Endl. ex Griseb. & $1300,2460,1813,1927$ & NA $(700-4350 \mathrm{~m})$ & LC \\
\hline Nertera granadensis (Mutis ex L. f.) Druce & $\begin{array}{l}\text { 1340, 1371, 1479, 2040, } \\
2421,1817,1705\end{array}$ & NA (1300-4300 m) & LC \\
\hline Palicourea angustifolia Kunth & $\begin{array}{l}1859,2097,2150,2328 \\
2399\end{array}$ & $\begin{array}{l}\text { NA, Guayana, Macarena, } \\
\text { SN San. Marta, Valle del } \\
\text { Magdalena (500-3600 m) }\end{array}$ & LC \\
\hline Palicourea aschersonianoides (Wernham) Steyerm. & $\begin{array}{l}1321,1341,1375,1802, \\
1877,2073,2080,2204, \\
2390\end{array}$ & $\begin{array}{l}\text { NA, Valle del Magdalena } \\
\qquad(800-3750 \mathrm{~m})\end{array}$ & NE \\
\hline
\end{tabular}


Appendix: Continuation.

\begin{tabular}{|c|c|c|c|}
\hline & $\begin{array}{c}\text { Voucher } \\
\text { P.A. Gil-Leguizamón }\end{array}$ & $\begin{array}{c}\text { Status/Biogeographical } \\
\text { Region }\end{array}$ & $\begin{array}{c}\text { IUCN Red List } \\
\text { Status }\end{array}$ \\
\hline Palicourea cf. amethystina (Ruiz \& Pav.) DC. & 2157,1677 & $\begin{array}{l}\text { NA, Valle del Magdalena } \\
(300-3800 \mathrm{~m})\end{array}$ & LC \\
\hline Palicourea demissa Standl. & 1570,2003 & $\begin{array}{l}\text { NA, SN San. Marta, Valle del } \\
\text { Magdalena (760-3750 m) }\end{array}$ & LC \\
\hline Palicourea gachetensis M.C.G. Kirkbr. & $2106,2391,2420$ & ECO $(2000-2880 \mathrm{~m})$ & $\mathrm{NE}$ \\
\hline Palicourea sp. & $2120,2217,2255,2501$ & - & - \\
\hline Psychotria sp. & 2333 & - & - \\
\hline \multicolumn{4}{|l|}{ Rutaceae } \\
\hline Zanthoxylum cf. quinduense Tul. & 2131 & NA $(1000-3300 \mathrm{~m})$ & $\mathrm{NE}$ \\
\hline \multicolumn{4}{|l|}{ Salicaceae } \\
\hline Abatia parviflora Ruiz \& Pav. & 1564 & NA (1990-3382m) & LC \\
\hline \multicolumn{4}{|l|}{ Santalaceae } \\
\hline Dendrophthora cf. costaricensis Urb. & 2570 & NA $(2300-3130 \mathrm{~m})$ & NE \\
\hline Dendrophthora cf. obliqua (C. Presl) Wiens & 1593 & $\begin{array}{l}\text { NA, Guayana, Macarena, } \\
\text { SN San. Marta, Valle del } \\
\text { Cauca, Valle del Magdalena } \\
\text { (400-2915 m) }\end{array}$ & NE \\
\hline Dendrophthora clavata (Benth.) Urb. & 1585,1643 & $\begin{array}{l}\text { NA, SN San. Marta (1940- } \\
\qquad 3900 \mathrm{~m})\end{array}$ & NE \\
\hline Dendrophthora lindeniana Tiegh. & $2088,2302,2304$ & $\begin{array}{l}\text { NA, SN San. Marta (1500- } \\
3350 \mathrm{~m})\end{array}$ & NE \\
\hline \multicolumn{4}{|l|}{ Solanaceae } \\
\hline Cestrum humboldtii Francey & $1565,1654,2002,2440$ & NA (1650-3200 m) & LC \\
\hline Cestrum tubulosum Sendtn. & $1839,2300,1679$ & $\begin{array}{c}\text { Nativ from Amazonas, } \\
\text { Guayana, Macarena, } \\
\text { Orinoquía }\end{array}$ & NE \\
\hline Deprea orinocensis (Kunth.) Raf. & 2430 & NA (1385-3300 m) & NE \\
\hline Dunalia trianaei Dammer & 1576 & From Colombia, Ecuador & EN \\
\hline Sessea cf. elliptica Francey & 2400 & $\begin{array}{l}\text { EA, from Ant, Nar, Put, Tol } \\
(1800-2880 \mathrm{~m})\end{array}$ & LC \\
\hline Solanum laevigatum Dunal & 2439 & $\begin{array}{l}\text { NA, Valle del Cauca (920- } \\
\qquad 3700 \mathrm{~m})\end{array}$ & NE \\
\hline Solanum nudum Dunal & 2115,2214 & $\begin{array}{c}\text { NA, Amazonia, Orinoquia, } \\
\text { Pacífico, SN San. Marta, } \\
\text { Valle del Cauca, Valle del } \\
\text { Magdalena (0-2670 m) }\end{array}$ & LC \\
\hline \multicolumn{4}{|l|}{ Symplocaceae } \\
\hline Symplocos sp. 1 & 2086 & - & - \\
\hline Symplocos sp. 2 & 2259 & - & - \\
\hline Symplocos theiformis (L. f.) Oken & $\begin{array}{l}1890,2163,2362,2477 \\
2563,1910\end{array}$ & $\begin{array}{l}\text { End. from Colombia (2450- } \\
\qquad 3724 \mathrm{~m} \text { ) }\end{array}$ & LC \\
\hline Symplocos venulosa Cuatrec. & 2068 & ECO $(2200-2950 \mathrm{~m})$ & VU \\
\hline
\end{tabular}


Appendix: Continuation.

\begin{tabular}{|c|c|c|c|}
\hline & $\begin{array}{c}\text { Voucher } \\
\text { P.A. Gil-Leguizamón }\end{array}$ & $\begin{array}{c}\text { Status/Biogeographical } \\
\text { Region }\end{array}$ & $\begin{array}{l}\text { IUCN Red List } \\
\text { Status }\end{array}$ \\
\hline \multicolumn{4}{|l|}{ Theaceae } \\
\hline Gordonia cf. fruticosa (Schrad.) H. Keng & 2064,2111 & $\begin{array}{l}\text { NA, SN San. Marta (1000- } \\
\qquad 3600 \mathrm{~m})\end{array}$ & LC \\
\hline \multicolumn{4}{|l|}{ Tropaeolaceae } \\
\hline Tropaeolum sp. & 1964 & - & - \\
\hline \multicolumn{4}{|l|}{ Urticaceae } \\
\hline Pilea smithii Killip & $\begin{array}{l}1346,1540,1863,1983, \\
2331\end{array}$ & $\begin{array}{l}\text { From Colombia in Boy, Cho, } \\
\text { Cun }\end{array}$ & NE \\
\hline \multicolumn{4}{|l|}{ Violaceae } \\
\hline Viola stipularis Sw. & 2278 & $\begin{array}{l}\text { NA, SN San. Marta (1900- } \\
\qquad 3240 \mathrm{~m})\end{array}$ & NE \\
\hline \multicolumn{4}{|l|}{ Vitaceae } \\
\hline Cissus colombiensis Lombardi & 2114 & NA (1000-1980 m) & NE \\
\hline
\end{tabular}

